\title{
Speed in Clinical Magnetic Resonance
}

\author{
Val M. Runge, MD, Johannes K. Richter, MD, and Johannes T. Heverhagen, MD, PhD
}

\begin{abstract}
The relevant clinical accelerated magnetic resonance techniques that are available currently for routine patient examinations are reviewed, presenting and discussing the benefits therein when compared with more conventional scans. The focus is on clinical use and practicality, with the review divided into 3 sections. Improvements in 3-dimensional acquisition are first discussed, specifically controlled aliasing in parallel imaging results in higher acceleration, related radial techniques, and CAIPI-Dixon-TWIST-VIBE. Simultaneous multislice imaging is then reviewed, focusing on current implementation for 2-dimensional imaging, including both echo-planar and fast spin echo techniques. The final topic is that of sparse reconstruction (data sparsity), discussing the principles therein, challenges, and current applications. Guidance in terms of clinical use for accelerated techniques is provided, focusing on enabling faster and improved clinical scan results.
\end{abstract}

Key Words: image enhancement, image processing, magnetic resonance angiography, magnetic resonance imaging, methods, signal processing, computer-assisted

(Invest Radiol 2017;52: 1-17)

$\mathrm{M}$ agnetic resonance imaging (MRI) is more time-consuming when compared with other diagnostic imaging techniques such as computed tomography or ultrasound. On the other hand, MRI provides information not available by other techniques and is critical for clinical decision-making. In the years since its introduction, in the early 1980s, technological advancements have significantly accelerated MRI, largely alleviating the aforementioned disadvantage. Much has happened since the early clinical advent of MR, when a single scan technique (and that at low resolution) required more than 40 minutes for acquisition ${ }^{1}$ and the importance of negating the effect of breathing had just been recognized. ${ }^{2}$ Building upon the innovations in the last 3 decades, future developments involving speed promise to improve MR even further, including specifically its clinical applicability and the reliance by clinicians on MR for patient diagnosis and evaluation.

Past and possible future developments will be discussed, focusing on the topic of speed, with reference to relevant enhancements in clinical care. The initial section describes "the way we came." Innovations important to speed are described briefly in historical order. The fundamental basis making this era possible was the development of parallel imaging. ${ }^{3}$ Generalized autocalibrating partially parallel acquisitions (GRAPPA) ${ }^{4}$ and controlled aliasing in parallel imaging results in higher acceleration (CAIPIRINHA) ${ }^{5}$ then appeared, with high radiofrequency (RF) coil density enabling full use of these techniques. High temporal resolution imaging, specifically TRICKS (time-resolved imaging of contrast kinetics), ${ }^{6,7}$ appeared slightly earlier, with subsequent

Received for publication August 9, 2016; and accepted for publication, after revision, September 9, 2016.

From the Department of Diagnostic, Interventional and Pediatric Radiology, Inselspital, Bern University Hospital, University of Bern, Bern, Switzerland.

Correspondence to: Val M. Runge, MD, Department of Diagnostic, Interventional and Pediatric Radiology, Inselspital, Bern University Hospital, University of Bern, Bern, Switzerland.

Conflicts of interest and sources of funding: Partial support was provided by an unrestricted educational grant from Siemens Healthineers.

The authors report no conflicts of interest.

The images illustrated in this review article were acquired on 1.5 and 3 T Siemens clinical MR systems.

Drs Runge and Richter are co-first authors.

Copyright (C) 2016 Wolters Kluwer Health, Inc. All rights reserved.

ISSN: 0020-9996/17/5201-0001

DOI: $10.1097 /$ RLI.0000000000000330 development of the technique time-resolved imaging with stochastic trajectories (TWIST). ${ }^{8}$ These approaches have been integrated with GRAPPA and CAIPIRINHA, making possible developments such as CAIPI-Dixon-TWIST-VIBE. ${ }^{9}$ Additional advances enabled radial imaging, allowing a further reduction in motion artifacts. For example, using radial volumetric interpolated breath-hold examination (VIBE), motion artifacts present as radial streaks or mild blurring, in distinction to the dominant ghost artifacts present with conventional rectilinear $\mathrm{k}$-space sampling. This approach allows for free-breathing examinations, with image quality similar to breath-hold rectilinear acquisitions. ${ }^{10}$ As a consequence, more patients can benefit from MR, because many are challenged by the breath-hold examination required for body imaging. Techniques permitting the clinical implementation of simultaneous multislice (SMS) imaging ${ }^{11}$ most recently have become available, with initial applications in the brain and for echo-planar imaging (EPI) in general.

The subsequent 2 sections of this review cover "the way ahead." Simultaneous multislice imaging is being further refined, and more importantly extended to techniques beyond EPI, with widespread clinical application evident. ${ }^{12}$ Sparse imaging, also discussed in detail, is the other major development allowing acceleration of the MR examination, further expanding the clinical utility and application of MR. ${ }^{13}$

\section{THE WAY WE CAME}

\section{Parallel Imaging}

In the early 1990s, multicoil arrays were introduced in MR for improved signal-to-noise ratio (SNR). ${ }^{14}$ These replaced the previous generation of volume and surface coils, which was composed of either 1 or 2 wire loops. This enabled the subsequent development of parallel imaging, first introduced in the late 1990s, which further revolutionized the field of MR. ${ }^{3,15,16}$ In these early articles, the use of multiple receiver coils in parallel was first shown, enabling scan time to be reduced. Central to this approach was the use of arrays of multiple receiver coils and the knowledge that receiver sensitivity could be used for encoding. As demonstrated, in its most basic implementation, scan time could be reduced by half using a 2-coil array. A primary limitation, however, to sensitivity encoding (SENSE), an early parallel imaging approach, was the requirement of accurate knowledge of the sensitivities of the component coils. This may be difficult to determine due to noise, with coil or subject motion between coil calibration and scan acquisition also being problematic. In 2002, the concept of parallel imaging was further advanced with the introduction of GRAPPA. ${ }^{4}$ In this approach, a detailed, accurate RF field map is not needed before image reconstruction. Obtaining a few additional k-space lines during image acquisition instead provides this information. The autocalibrating nature of GRAPPA enables improved parallel imaging in instances where acquired coil sensitivity maps may be in error, which occurs with motion (e.g., abdominal and cardiac imaging) and with single-shot EPI (ss-EPI) in areas of severe distortion (e.g., in brain imaging near the paranasal sinuses). Since image reconstruction and image combination are performed in separate steps, additional important results include higher SNR and improved image quality.

Parallel imaging, as described in the previous paragraph, thus allows increased imaging speed, a major reason for its development. The increase in speed is obtained by reducing the number of required phase encoding steps. This is accomplished by undersampling $\mathrm{k}$-space, yet keeping the maximum $\mathrm{k}$ values to preserve spatial resolution. 
Unfortunately, parallel imaging is intrinsically associated with a loss in SNR, due to the reduction in number of data samples acquired. With parallel imaging applied, SNR is proportional to $1 / \sqrt{ } \mathrm{R}(\mathrm{R}$ being the acceleration factor). ${ }^{15}$ Applying an acceleration factor of 2 results in a reduction of SNR by $\sqrt{2}$, whereas applying a factor of 4 results in a 2-fold reduction in SNR. Thus, the applications of parallel imaging, when applied by itself, are generally restricted to high SNR images. One exception is single-shot diffusion-weighted imaging, where the application of parallel imaging (used to reduce bulk susceptibility artifacts) shortens the signal readout with the increase in SNR therein partially compensating for the loss due to reduction in number of phase encoding steps.

\section{CAIPIRINHA}

In 2006, 2-dimensional (2D) CAIPIRINHA was introduced. ${ }^{5}$ The group from Würzburg demonstrated that a reduction in the required data (parallel imaging) could be accomplished for 3-dimensional (3D) imaging simultaneously in 2 spatial dimensions (thus the name 2D CAIPIRINHA). By modifying the phase encoding sampling strategy, aliasing is shifted in a way that exploits the underlying receiver array more efficiently. The result is improved image quality, with a more robust and homogenous reconstruction. Two-dimensional CAIPIRINHA is applicable to all commonly used imaging techniques.

By 2013, 2D CAIPIRINHA had been implemented for clinical use, with the Mannheim group demonstrating comparable image quality using an acceleration factor of $\mathrm{R}=2 \times 2$ with VIBE for the abdomen when compared with an $\mathrm{R}=2$ GRAPPA acceleration. ${ }^{17}$ Acquisition time was reduced from 21 to 9 seconds for a $320 \times 175$ matrix. By allowing shorter breath-hold acquisition times, image quality is more robust, with imaging also made possible in patients that cannot comply with long (20 seconds) breath-hold requirements. A subsequent study in 2014 used CAIPIRINHA to reduce the overall acquisition time from 20 to 13 seconds to reduce the detrimental effect of respiratory motion during the hepatic arterial phase for gadoxetic acid-enhanced studies. With this approach, the number of nondiagnostic arterial phase scans was reduced, and image quality improved in general for the hepatic arterial phase. ${ }^{18}$ Although there are many different ways to achieve shorter breath-hold scans, it is clear that this general approach will improve patient compliance and lead to higher diagnostic quality in abdominal MRI (Fig. 1).

A further evolution of this approach is CAIPIRINHA-DixonTWIST (CDT) VIBE. This highly accelerated T1-weighted sequence enables time-resolved imaging of the abdomen during and immediately after bolus intravenous contrast administration. Parallel imaging, undersampling, view sharing, and Dixon water-fat separation are combined. Use of the Dixon-based fat separation technique results in improved SNR on reconstructed water-only images. In the initial clinical implementation, fourteen 3D data sets of the liver were acquired during the arterial phase, starting 5 seconds after contrast administration, with a 29 -second breath-hold. The temporal resolution was 2.6 seconds, and the temporal footprint was 6.2 seconds. ${ }^{9}$ There was added diagnostic value in $21 \%$ of patients with CDT-VIBE. In this small patient series, 2 patients (of 47 total) also demonstrated hypervascular lesions (presumed metastases) that were visualized only on the CDT-VIBE scans. A disadvantage of this technique, however, is the large number of images that are acquired (nearly 1000 during a 29 -second period, just
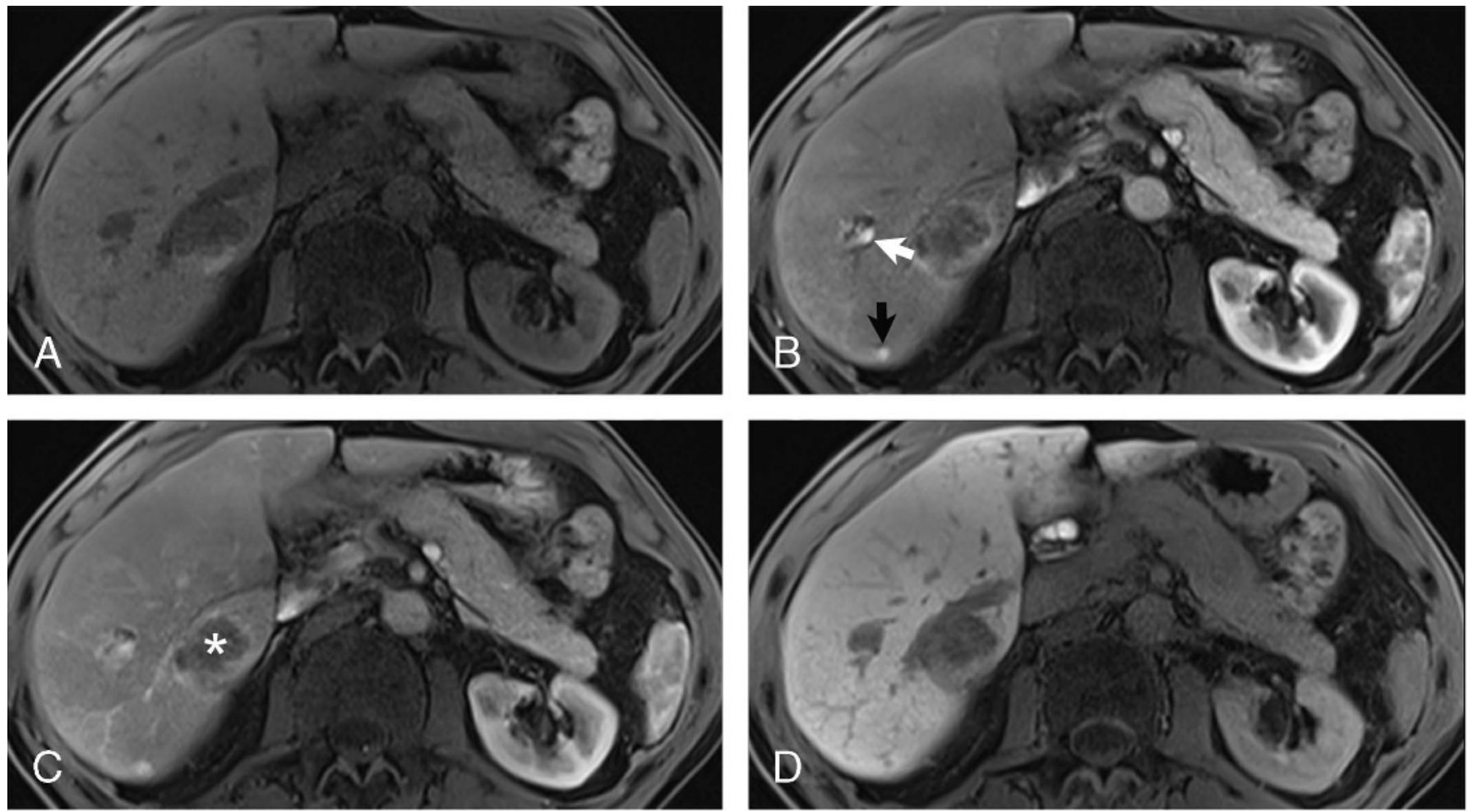

FIGURE 1. Application of an ultrashort breath-hold Dixon VIBE sequence for improved arterial phase imaging, with resultant overall improved image quality due to better patient compliance. Precontrast (A), first (B), and third (C) arterial phase images, and 20-minute delayed hepatocellular phase images (D) after injection of gadoxetic acid are presented in a 74-year-old man with metastatic rectal adenocarcinoma. Acquisition time for each image series was 4.8 seconds, with scans acquired at 3 T using a 60-channel body coil. A new small metastasis along the dorsal margin of the liver (black arrow) demonstrates prominent early arterial enhancement, with some wash out by the late arterial image, and no uptake in the delayed phase. Also visualized at this level are a hemangioma (white arrow) and a larger metastasis previously treated by microwave ablation (asterisk). Note the absence of respiratory artifacts due primarily to the short scan time, as compared with more conventional 15 to 20 seconds VIBE acquisitions. 
considering the different anatomic slices and temporal time frames). Because of the time needed for image review if the examination were viewed in a conventional fashion, implementation in routine clinical practice will require advanced viewing strategies and/or dedicated 4-dimensional software. A subsequent study evaluated, for detection of focal liver lesions, CDT-VIBE with 5 hepatic arterial subphases in 24 patients. In one third of cases, the multiarterial image acquisition improved the lesion detection rate when compared with the equivalent standard arterial phase, in particular, for hypervascular lesions. ${ }^{19}$ The arterial parenchymal contrast was also better, in 19 of 24 patients, on one of the multiarterial image acquisitions when compared with the reference standard.

CAIPIRINHA-Dixon-TWIST-VIBE has also been exploited to provide higher temporal resolution, while preserving diagnostic image quality, in dynamic contrast-enhanced MRI of the prostate. ${ }^{20}$ This approach provides high diagnostic accuracy, through improved parameter modeling, for the discrimination between benign and malignant lesions. ${ }^{21}$

Critical to the implementation of these and subsequently described techniques was the concurrent development of advanced coil designs, with increased number of coil elements and higher coil density. Current designs feature, for example, 64 channels for a head/neck coil and 60 channels for a body coil. This requirement was noted in early articles describing GRAPPA ${ }^{4}$ and subsequently CAIPIRINHA. ${ }^{5}$ Magnetic resonance systems with a very high number of channels make certain applications feasible and also provide more general benefits in terms of higher SNR and in the application of parallel imaging. Likewise critical for clinical utilization of these advanced techniques were improvements in hardware for image reconstruction, including both computational speed and storage.

\section{Radial Imaging}

In 2011, radial sampling of $\mathrm{k}$-space with VIBE became available, with initial evaluation in the liver (for contrast-enhanced fat-suppressed T1-weighted imaging). The implementation of radial VIBE used conventional k-space sampling in the slice direction and radial sampling in-plane ("stack of stars"). Comparison of radial VIBE obtained in free-breathing to conventional breath-hold VIBE for contrast-enhanced liver imaging revealed comparable image quality (Fig. 2). However, the initial implementation of this sequence led to scan times on the order of a minute, as compared with a typical breath-hold acquisition time for rectilinearly sampled k-space of 14 seconds. So, in its initial implementation, although radial VIBE produced equivalent image quality, without requiring breath-holding, the sequence was not suitable for implementation with dynamic imaging, due to the long acquisition time. ${ }^{10}$

Subsequent evaluation for pediatric imaging compared radial (free-breathing) and Cartesian (breath-hold when possible) VIBE, with radial VIBE demonstrating better image quality and lesion conspicuity. More lesions were detected on the radial scan than on Cartesian VIBE. ${ }^{22}$ On this basis, radial VIBE is recommended for abdominopelvic MR examinations in pediatric patients, an important patient population in which acquisition of scans during breath-holding can be challenging.

Other anatomic areas can also benefit from use of radial VIBE, due to motion of the anatomic structures therein. As with the liver, imaging of the pancreas can similarly be done for patients who cannot hold their breath. ${ }^{23}$ Given the importance of contrast enhancement with fat suppression in the orbit, and the inherent motion of the globe that occurs during any scan, this area is an excellent one for application of a radial VIBE sequence. Superior image quality has been demonstrated, regardless of parameter used for assessment, including overall motion artifact, depiction of intraorbital vessels, extraocular muscles, and optic nerves, and evaluation of pathology. ${ }^{24}$ Imaging of the soft tissues of the neck is commonly degraded due to swallowing, breathing, and inadvertent patient motion, with contrast-enhanced radial VIBE scoring
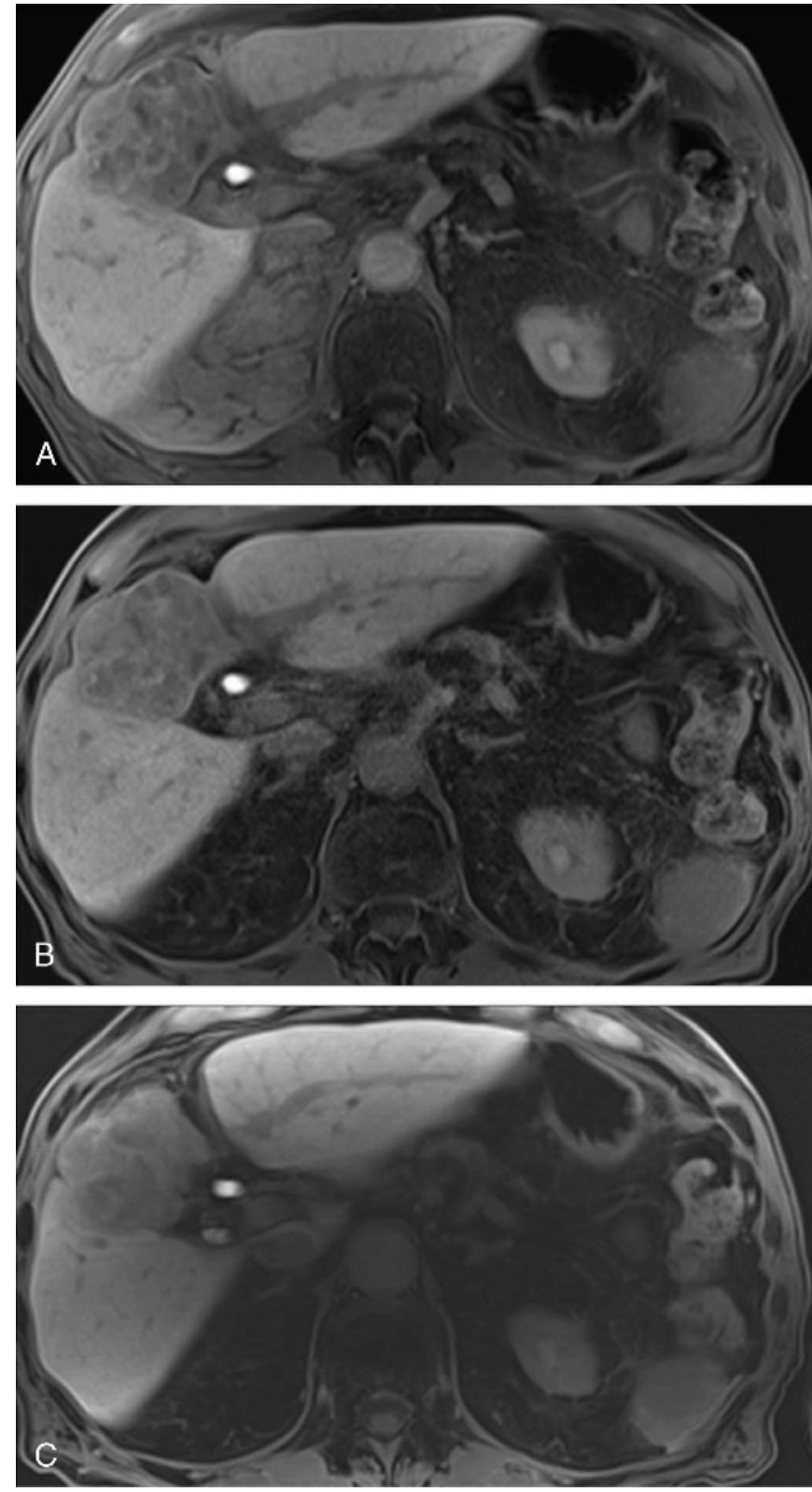

FIGURE 2. Comparison of (A and B) breath-hold and (C) non-breath-hold imaging for the liver. Conventional 16-second VIBE $(A)$ and 4.8-second Dixon VIBE (B) acquisitions are compared with a 2-minute radial VIBE (C) acquisition. All are from imaging in the hepatocellular phase at 20-minutes after injection of gadoxetic acid, in an 80-year-old male patient with hepatocellular carcinoma (HCC) in segments IV/V/VIII being treated with sorafenib. Scans were acquired at 3 T using a 60-channel body coil. Note the difference in the signal intensity of fat ( $A$ vs $B$ and $C$ ), due to the different approaches used for fat suppression. Diagnostic information in the 4.8-second acquisition is equivalent to that in the 16-second acquisition, both breath-hold. In addition, despite acquisition during free-breathing (C), fine anatomic detail within the liver, for example, the vasculature of the left lobe, is well depicted using the radial VIBE technique.

significantly better than conventional VIBE in all evaluated image quality measures in this application as well. ${ }^{25}$ For the head and neck, radial VIBE represents a motion-robust improvement over a conventional VIBE acquisition. Radial VIBE and its variants have been evaluated at both 1.5 and $3 \mathrm{~T}$, with utility regardless of field strength. 

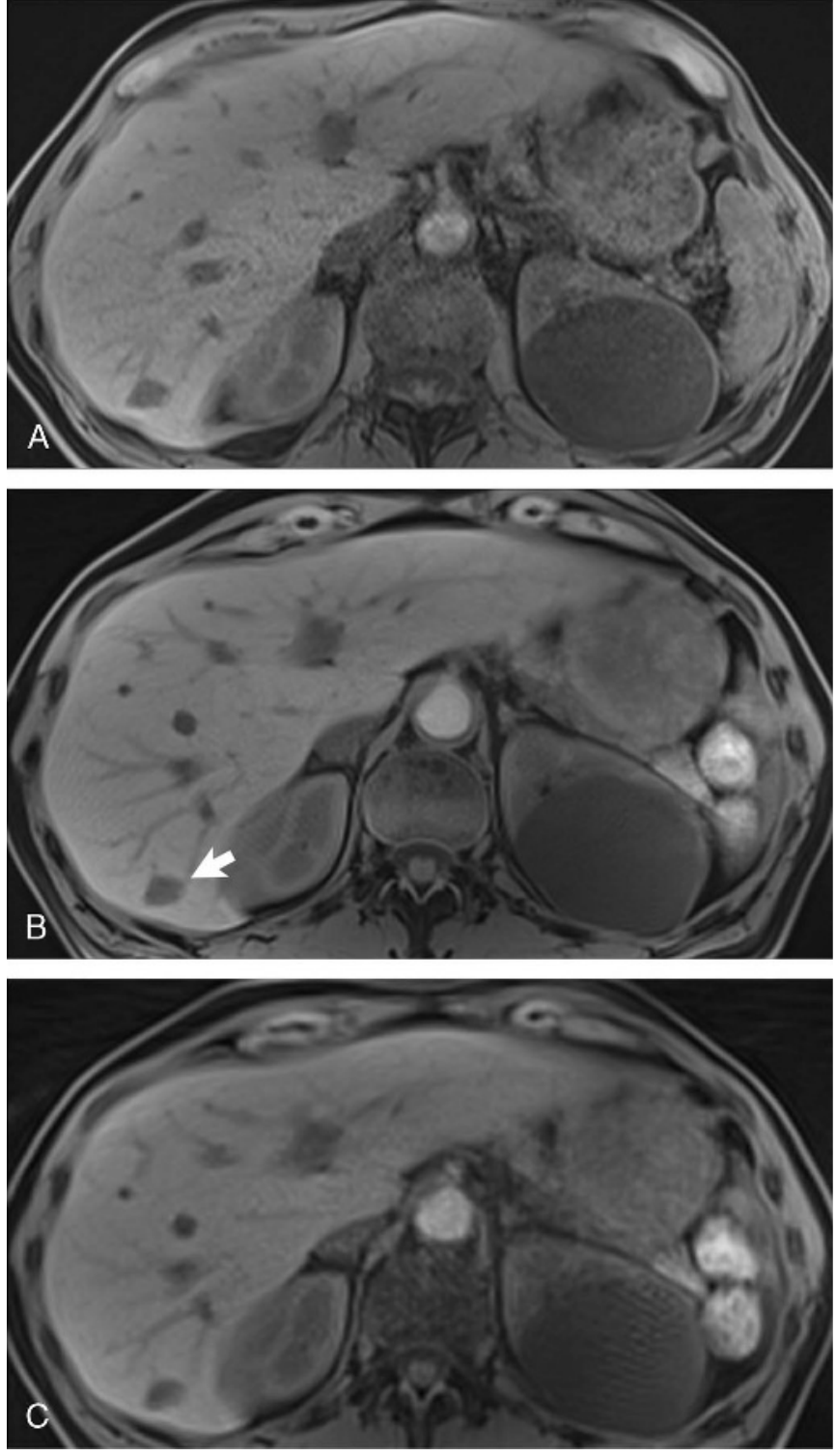

FIGURE 3. Speed in MR, how to proceed? Two radically different approaches to T1-weighted imaging in the liver are compared, an ultrashort breath-hold Dixon VIBE sequence $(A)$ versus non-breath-hold radial VIBE ( $B$ and $C$ ) scans. All images were acquired at $3 \mathrm{~T}$ using a 60 -channel body coil, with a slice thickness of $3 \mathrm{~mm}$, and without contrast administration. A large left simple renal cyst is noted, together with a single smaller cyst (arrow) within the liver. In panel A, the scan sequence was optimized to enable a 4.8-second acquisition, taking advantage primarily of the SNR of the advanced design, high element number, body coil. Scan times for panels B and C were 2 minutes versus 21 seconds, with the latter optimized for a shorter scan time principally by restricting the number of radial views (680 vs 180 ).

Subsequent to its initial development, radial VIBE became one of the early pulse sequences successfully implemented in combination with compressed sensing. In a volunteer study, comparing breath-hold VIBE to free-breathing golden-angle radial sparse parallel (GRASP, the radial compressed sensing implementation of VIBE), with acquisition of arterial and venous phase images, GRASP was shown to have acceptable image quality, although with lower overall image quality. ${ }^{26}$

It should be clear from the prior discussion, as well as from subsequent sections, that speed in MR can mean many things. This ranges from the obvious of shorter scans to the ability to acquire advanced scan techniques within a clinically acceptable acquisition time (Fig. 3).

\section{THE WAY AHEAD}

\section{Simultaneous Multislice Imaging}

\section{Theory}

When MR was initially introduced in the early 1980s, acquisition of only a single slice was possible. ${ }^{27}$ Rapidly thereafter, 3D techniques were developed, ${ }^{28}$ and subsequently $2 \mathrm{D}$ multislice imaging. ${ }^{29}$ The latter represented a major advance for clinical application, providing multiple images in the same time previously required for a single slice. Multislice imaging has since then become a mainstay for clinical MR, being used in essentially every clinical 2D acquisition. The name "multislice," however, can be somewhat misleading. With this technique images are not actually acquired simultaneously, but rather each slice is acquired sequentially within a very short span of time. True SMS, also known as multiband, imaging was conceptualized much later, ${ }^{30,31}$ with a viable approach for clinical implementation presented only recently (Fig. 4). ${ }^{11,32,33}$ Simultaneous multislice represents likely the single most significant advance for clinical MR in the current decade, making possible in its initial implementation scan time to be further reduced by a factor of 2, and in certain instances by up to a factor of 3 to 4 . It should be noted that implementation of SMS would not be possible without substantial prior advances in both hardware and software, including multicoil arrays, CAIPIRINHA, ${ }^{34}$ and slice-GRAPPA reconstruction.

The interest in the use of SMS is based upon the increase in temporal efficiency that can be achieved with this parallel imaging-based sequence. The availability of SMS has already fundamentally changed the scope of studies that can be realistically acquired clinically both with echo-planar and fast spin echo (SE) sequences, as will be discussed in subsequent sections. Equally or perhaps more important in the current clinical environment is the application of SMS to shorten scan times and improve patient throughput.

\section{Diffusion-Weighted Imaging}

For diffusion-weighted scans, rapid single-shot 2D SE EPI sequences are commonly used. As conventionally implemented, 2D SE EPI is highly inefficient time-wise, because diffusion encoding, which represents a significant portion of the acquisition time, is performed for the whole imaging volume with each single 2D imaging slice excitation. Parallel imaging can be, and typically is, used. However, the application of parallel imaging in this instance does not provide significant acceleration to the scan, because it only shortens the EPI encoding period. The use of parallel imaging in this application is important, regardless, for reducing image distortion and blur. Simultaneous multislice on the other hand allows for concurrent acquisition of multiple slices, with scan time reduced by the slice acceleration factor (mitigated to a small extent by the time needed for the fast reference scan, the latter required for slice separation during image reconstruction). Unlike with parallel imaging by itself, there is no SNR penalty related to the acceleration, which with parallel imaging occurs due to acquisition of a reduced number of phase encoding lines (SNR being proportional to $1 / \sqrt{ } \mathrm{R}$, where $\mathrm{R}$ is the acceleration factor). Both SMS and traditional parallel imaging, however, suffer from g-factor-related SNR loss, which for both cases is closely related to coil geometry and overall acceleration factor.

Critical to the implementation of SMS is the unaliasing of the simultaneously acquired yet closely spaced slices. Use of a blipped CAIPIRINHA approach allows sufficient interslice image shifts between aliased voxels in the phase encoding direction, avoiding the high g-factor (SNR) penalty and blurring ("tilted voxel") artifact associated with previous approaches. ${ }^{11}$ Important as well has been the implementation of specialized reconstruction techniques to reduce the leakage 


\section{Simultaneous Multi-Slice}

Simultaneous excitation of multiple slices with blipped CAIPIRINHA ${ }^{1}$
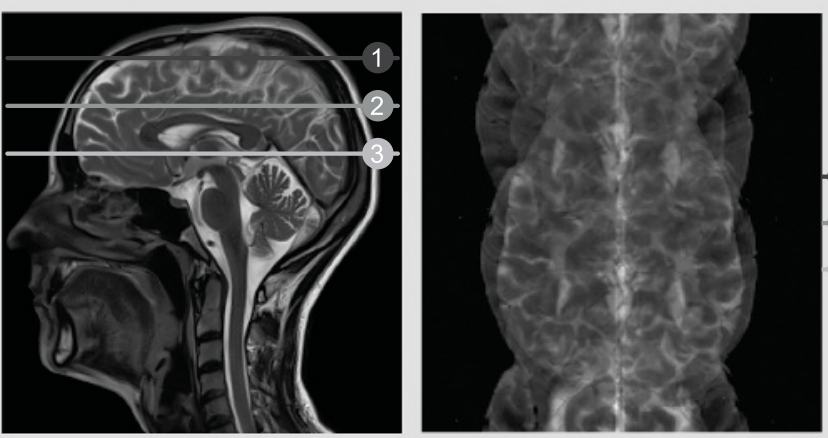

Multiple slices excited simultaneously

Blipped CAIPIRINHA
applied during echo train
Minimization of g-factor
related SNR loss

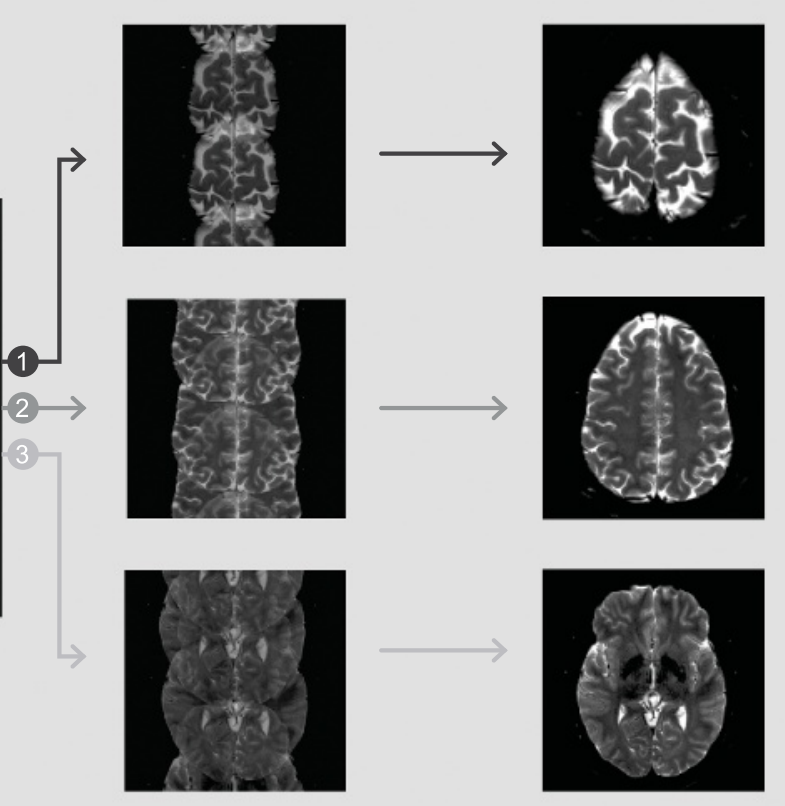

Slice GRAPPA

based unaliasing
Inplane GRAPPA

based unaliasing

FIGURE 4. Diagrammatic representation of slice acquisition and reconstruction using SMS, specifically with excitation of multiple slices by using blipped CAIPIRINHA followed by slice and then in-plane unaliasing both using GRAPPA. Figure 4 can be viewed online in color at www.investigativeradiology.com.

signal contamination between simultaneously acquired slices. ${ }^{35}$ Another consideration is the minimization of the Nyquist (N/2) ghosting artifacts unique to EPI. For example, depending on the specific scan, a ghost artifact from 1 slice in SMS-EPI might overlie directly the center of the imaging FOV of another imaging slice.

Of particular importance for the application of SMS in diffusionweighted imaging is the design of the multiband RF pulse, which specifically allows for simultaneous excitation and refocusing of multiple slices. Application of such multiband RF pulses increases specific absorption rate (SAR). At $3 \mathrm{~T}$, use of low SAR variable-rate selective excitation (VERSE) pulses ${ }^{36}$ - introduced in the early 2000 s and subsequently widely used at $3 \mathrm{~T}$-provides adequate SAR reduction. Further attention to slice profile issues is required in this application, due to the implementation of VERSE. Other approaches have been suggested as well to permit imaging without increased peak power or SAR. ${ }^{37,38}$

Subsequent to the development of SMS for ss-EPI, attention turned to the implementation of this technique for diffusion-weighted readout-segmented EPI (rs-EPI, RESOLVE). ${ }^{39}$ Although ss-EPI continues to be widely used, this technique suffers from geometric distortion and blurring, the latter due to T2* decay. These 2 factors limit the use of ss-EPI at high field strengths, where improved spatial resolution would be a major goal.

Readout-segmented EPI provides improved image quality for diffusion-weighted imaging in a wide range of clinical applications. Critical to this technique is the use of a navigator echo that acquires phase information after each shot to correct motion-induced phase errors, enabling reconstruction of an artifact free image. The navigator is also used for identification and reacquisition of shots with severe motion-induced phase artifacts. Geometric distortion and blurring are markedly reduced with rs-EPI compared with ss-EPI, due to the acquisition of subsets of k-space during multiple repetition times (TRs) rather than encoding the whole of k-space in a single shot. Visualization of areas prone to bulk susceptibility artifacts, particularly the interfaces between the paranasal sinuses and brain, is markedly improved. Reduced geometric distortion also impacts in a positive way assessment of lesion volume, which is important in brain ischemia for clinical evaluation, therapeutic decision planning and prognosis, and in other areas of the body with neoplastic disease for treatment planning. ${ }^{40,41}$ Another inherent advantage to rs-EPI is that the reduced duration of the EPI readout results in a shorter TE, reducing signal loss due to T2 decay.

An important limitation of rs-EPI, however, is that scan time is increased compared with ss-EPI. Thus techniques to accelerate the sequence are important for clinical use, not only for routine screening examinations but also for advanced applications such as DTI and tractography, with a major role for SMS. As previously noted, data from multiple slices are acquired simultaneously with SMS. ${ }^{42}$ With fewer slice excitations needed to cover the anatomic volume, TR can be reduced and thus the scan accelerated.

At $3 \mathrm{~T}$ rs-EPI is the diffusion-weighted technique preferred by neuroradiologists for the diagnosis of brain ischemia and the recognition of tumor hypercellularity, with ss-EPI less commonly used 

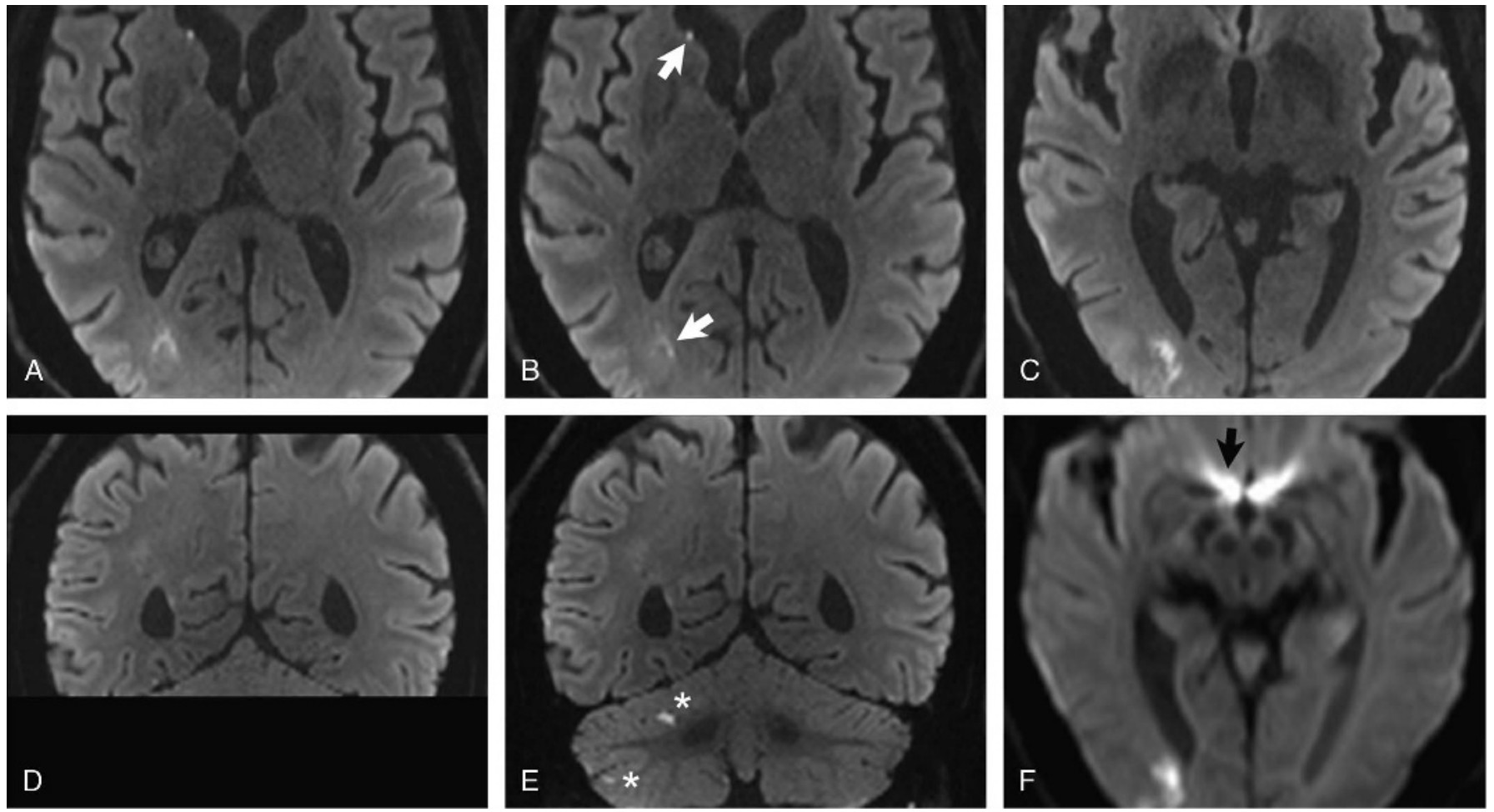

FIGURE 5. Single-shot diffusion-weighted $(b=1000)$ EPI demonstrating the use of SMS for increased anatomic coverage when using thin sections. Scans were acquired at $3 \mathrm{~T}$ using a 64-channel head and neck coil, in a 68-year-old male patient with multiple, small, bilateral subacute infarcts. Conventional (A) and SMS (B) (2x acceleration) acquisitions are compared, with 4:24 and 4:39 min:s scan times, respectively. In-plane resolution was $0.6 \times 0.6$ mm², with a slice thickness of $1 \mathrm{~mm}$. The 2 axial scans are equivalent (given minimal differences in slice position), both depicting a tiny right caudate head lacunar infarct and a small right parietal watershed infarct (arrows). Coronal reformatted images (D, conventional; $E, S M S)$ from the 2 respective examinations demonstrate the increased slice coverage with SMS, allowing depiction of the cerebellum and 2 small lacunar infarcts (asterisk) therein. The SMS acquisition is also compared with a thick section $(5 \mathrm{~mm})$ conventional DWI scan (C vs F), at the level of the brainstem, demonstrating the marked blurring and prominent susceptibility artifacts (black arrow) inherent with the latter approach. (Images acquired with the assistance of William Almiri, Bern University Hospital).

clinically for this purpose. This shift occurred before the development of SMS, despite the relatively long scan times for rs-EPI (3-5 minutes) when compared with ss-EPI (often on the order of 1 minute) when used for screening of the brain. Simultaneous multislice can be used in a simplistic way in routine clinical examinations to reduce the acquisition time for rs-EPI, or it can be used in a more elegant fashion to make possible higher resolution scans not previously feasible (due to long scan times).

Outside of the brain, rs-EPI has many important applications. In each of these applications, SMS improves further the utility of the technique. Imaging of the cervical cord is markedly improved with rs-EPI, with SMS providing greater slice coverage for axial imaging. Because of prominent susceptibility artifacts, ss-EPI is not a viable option for imaging of the spinal cord. The head and neck (for evaluation of soft tissue tumors, and most importantly squamous cell carcinoma $)^{43}$ and the breast ${ }^{44,45}$ are other important areas of application for SMS rs-EPI

\section{Fast (Turbo) Spin Echo Imaging}

Simultaneous multislice can be applied to fast SE imaging, as has been done with EPI, although this development is more recent. The impact of SMS fast spin echo (FSE) sequences will likely be similar to that of DWI SMS sequences, substantial and across a breadth of anatomic applications. Immediate applicability would be to scans in which 2 or more concatenations are now needed, to obtain sufficient slice coverage. Additional attention may be needed to SAR considerations, specifically at $3 \mathrm{~T}$ as opposed to $1.5 \mathrm{~T}$. In terms of tissue contrast,
SMS scans with proton density-, T1-, and T2-weighting have all been evaluated, albeit with limited clinical experience.

\section{Clinical Studies/Applications}

\section{Diffusion-Weighted Imaging}

The implementation of SMS in DWI can take various different forms. More diffusion encoding directions can be obtained in a clinically relevant timeframe. This enables more complex diffusion approaches, with applications for fractional anisotropy, diffusion kurtosis, and fiber tracking. Simultaneous multislice can be applied to acquire a larger number of slices in the same scan time. In addition, by extension, this can facilitate the acquisition of thinner sections while maintaining anatomic coverage, without large time penalties. Furthermore, in its most simplistic implementation, it can be used to reduce the time for scan acquisition.

Brain. The utility of diffusion for evaluation of the brain is high, with many diverse applications. These include the diagnosis of acute infarction (and decisions regarding clinical management), detection of drug related cytotoxic edema (e.g., with methotrexate), differential diagnosis of tumors (with restricted diffusion characteristic, e.g., of an epidermoid, as well as CNS lymphoma), and tumor grading (with an astrocytoma). For current clinical examinations, diffusion trace and apparent diffusion coefficient maps are routinely acquired, with scan times on the order of 5 minutes or less (with scan times for ss-EPI much less than that for rs-EPI). With SMS, because multiple slices 
are excited simultaneously, the TR for the desired spatial coverage can be reduced. For example, a typical TR at $3 \mathrm{~T}$ using ss-EPI DWI for coverage of the whole brain in the axial plane (34 slices using a 4-mm slice thickness) is 6300 milliseconds. This can be reduced to 3500 milliseconds, without any substantial loss in SNR, by using an SMS acceleration factor of 2 , yet maintaining slice coverage. The decrease in scan time is directly proportional to the reduction in TR (not considering the small amount of time required for the additional reference scan). Simultaneous multislice has been implemented both for ss-EPI and multishot rs-EPI (RESOLVE). In the application with Ss-EPI, the resultant time savings is small given the shorter scan times involved (1-2 minutes), when used in the usual screening fashion at $3 \mathrm{~T}$ with thicker $(4 \mathrm{~mm})$ sections. However, in application for highresolution, thin section imaging, the time savings can be substantial (Fig. 5). This approach also has the advantage of decreasing (due to the small voxel size) bulk susceptibility artifacts, which limit the acceptance of ss-EPI for clinical brain imaging at $3 \mathrm{~T}$. Readoutsegmented EPI is commonly used in routine clinical examinations at $3 \mathrm{~T}$ due to reduced bulk susceptibility artifacts and image blur, but suffers from substantially prolonged scan times when compared with ss-EPI. In the immediate future, SMS in combination with EPI will likely represent the standard implementation of DWI for $3 \mathrm{~T}$ of the brain, whether used as a screening examination with thicker $(4 \mathrm{~mm})$ sections, achieving scan times on the order of 2 minutes (with rs-EPI), or applied for whole-brain thin section imaging, with clinically acceptable scan times of 4 to 6 minutes (with ss-EPI). The scan times provided are with an acceleration factor of 2 . Further time savings are achievable in routine clinical imaging at $3 \mathrm{~T}$ using 3-fold slice acceleration in combination with high-density coil arrays such as a 64-channel head and neck coil. However, further slice acceleration, for example, SMS 4, is likely not achievable without the application of specialized low-power RF pulses ${ }^{38}$ or other new approaches.

Diffusion tensor imaging (DTI) can be used to characterize white matter pathology (e.g., in multiple sclerosis with otherwise normal-appearing white matter) and to create tractography for the localization and evaluation of white matter pathways. Scans used for tractography are often 10 minutes or more in duration. Simultaneous multislice in this situation has been used to make tractography possible in routine clinical practice (due to shorter scan times), for example, for visualization of the corticospinal tract and arcuate fasciculus in patients with intra-axial neoplasms, specifically for neurosurgical planning. After acquisition, tractography and fMRI data are then transferred to the surgical navigation system, enabling tailoring of the neurosurgical approach. The improvement in quality of DTI with this reduction in scan time, leading to less inadvertent patient motion, should also not be underestimated.

Implementation of more advanced acquisition strategies for DTI, increasing the number of gradient directions, spatial resolution, or diffusion weightings, quickly leads to scans of 15 to 30 minutes duration (when acquired without SMS). This limits their use to volunteers or selected populations, specifically being impractical for sick patients. Simultaneous multislice can be used as well with such advanced acquisition strategies, making possible, for example, in more reasonable scan times, depiction of important thalamic substructures (e.g., in patients with Parkinson disease in late stages of the disorder, in preparation for deep brain stimulation electrode implantation) and brainstem anatomy. ${ }^{46}$ Furthermore, using SMS to allow sampling of more diffusion directions (within a reasonable scan time) can improve substantially the tracking of fiber pathways in areas of crossing fibers or cerebral edema (where fractional anisotropy is low).

Conventional EPI is widely used as well for functional imaging of the brain (BOLD). Multiple 2D slices are acquired in rapid sequence, with the sensitivity of the technique dependent upon the ratio between the size of the effect and the degree of noise. Repeated sampling in time provides sufficient SNR for detection of such brain activation. In typical
BOLD fMRI acquisitions, physiological noise is dominant, with a high degree of temporal autocorrelation, suggesting that there is little to be gained by an increase in imaging rate. Faster sampling in this instance would not necessarily increase the number of independent samples. However, acquisitions in which uncorrelated thermal noise is dominant

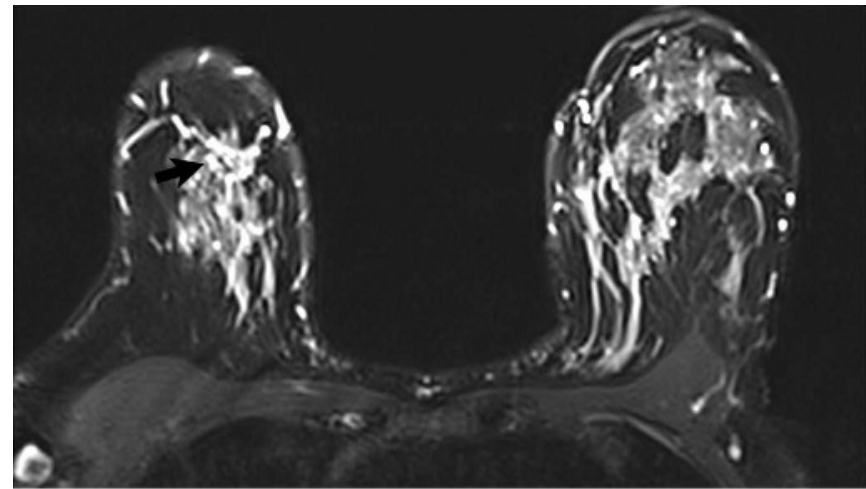

A

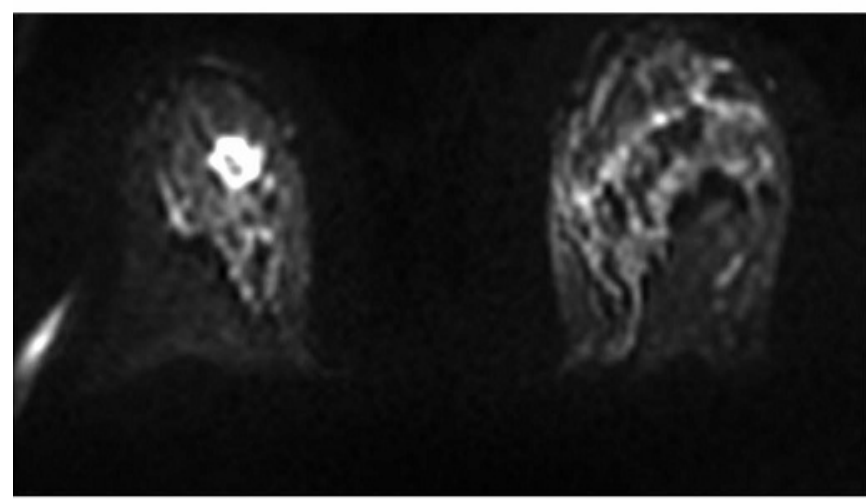

B

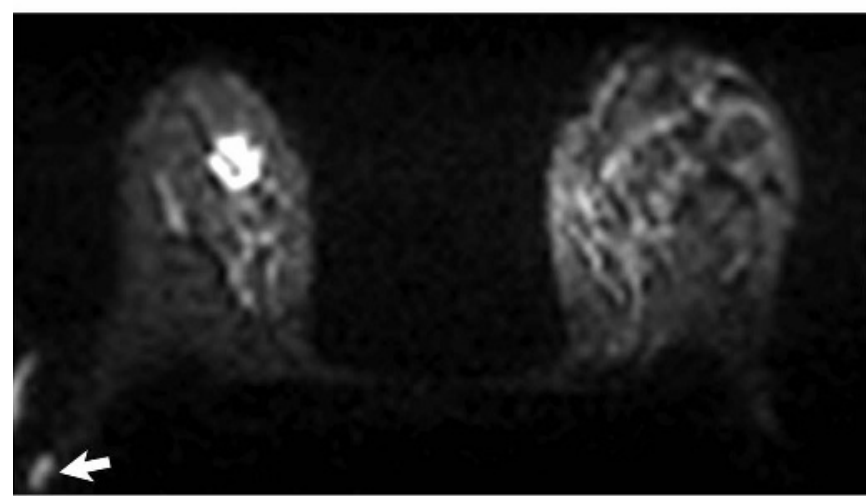

C

FIGURE 6. Application of rs-EPI DWI SMS in the breast for shorter scan acquisition and improved image quality. A spiculated, hyperintense lesion (black arrow) highly suspicious for malignancy is noted on the T2-weighted scan in the right breast in this 51-year-old woman (A). $B$, The conventional single-shot DWI sequence acquired with a selective field-of-view (zoomed), for faster speed and decreased susceptibility artifacts as well as geometric distortion, is compared with (C) the SMS ( $2 \times$ acceleration) rs-EPI diffusion-weighted scan. The tumor, with restricted diffusion (high signal intensity) is similarly depicted, with scan time decreasing from 5:52 to 3:17 min:s. On the rs-EPI scan, the metastatic lymph node in the right axilla (white arrow) can also be visualized, which is not depicted in panel $B$ due to residual susceptibility issues/geometric distortion. 

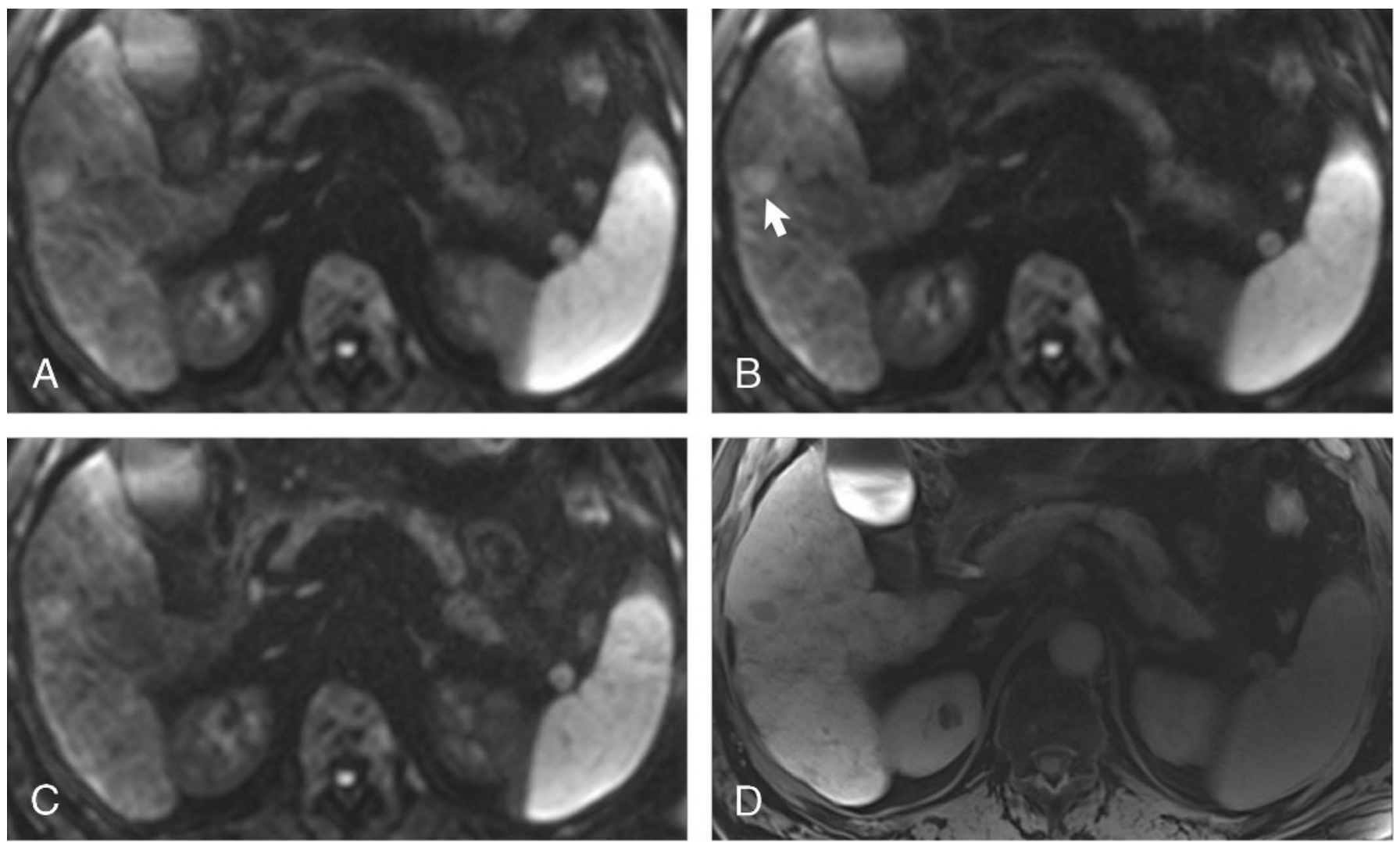

FIGURE 7. Application of 2D EPI DWI SMS in the liver for a shorter scan acquisition or alternatively thinner sections, with imaging at $3 \mathrm{~T}$. DWI scan times were (A) 2:41 min:s (non-SMS), (B) 1:38 min:s, and (C) 2:54 min:s (both SMS with a $2 \times$ acceleration). The slice thickness in panels A and B was equivalent $(5 \mathrm{~mm})$, with panel $C$ performed using a thinner slice $(3 \mathrm{~mm})$, and complete liver coverage with all scans. A small HCC with restricted diffusion (arrow) is noted in segment 5, in this patient with multifocal HCC. As illustrated, SMS in this instance can be used either to decrease scan time or to provide thinner sections with near equivalent image quality in a similar scan time. The lesion is also well seen on delayed imaging after gadoxetic acid administration (with no contrast uptake), using (D) a non-breath-hold 2-minute acquisition radial VIBE scan.

may benefit substantially from an increase in imaging rate. At $3 \mathrm{~T}$ this is true for both high spatial resolution BOLD and arterial spin labeling. For BOLD fMRI, a relatively long TE is needed for each slice to achieve sufficient $\mathrm{T} 2 *$-weighting (and thus sensitivity to blood oxygenation). The application of SMS in this circumstance allows preservation of the required long TE while reducing the time required to cover the entire brain. Extensive evaluation comparing scans utilizing no acceleration $(\mathrm{TR}=3 \mathrm{~s})$ versus a $6 \times$ acceleration $(\mathrm{TR}=0.5 \mathrm{~s})$, assessing both 2 and $3 \mathrm{~mm}$ scans, revealed a substantial improvement in sensitivity, specificity, and spatial detail with slice acceleration for BOLD fMRI. ${ }^{47}$ This improvement in speed of acquisition has broad applications, but in particular, clinically in instances where time is limited and repeated fMRI runs not possible, such as presurgical mapping. One restriction in early implementations of SMS for this and other applications was reconstruction time, which is no longer an issue using current clinical packages.

Resting state fMRI identifies networks of connectivity across the brain. As with the application of fMRI in specific tasks, SMS in resting state fMRI enables the sampling of more time points in a given duration (by the use of a shorter TR), increasing the degrees of freedom (in the appropriate circumstance decreasing noise), and thus improving statistical significance. The gains with SMS can be used in several ways to improve the quality of the resting state fMRI data. Smaller voxels can be achieved, or alternatively a shorter scan time, 2 typical applications of SMS. With higher temporal resolution, physiology-related signal changes from breathing and blood flow can also be more easily disentangled. Alternatively, SMS can be used in resting state fMRI to improve statistical significance, for example, allowing detection of more subtle differences between networks. ${ }^{48,49}$ Simultaneous multislice has been central to the Human Connectome Project (HCP) from the onset, with HCP focusing on characterizing connectivity in the brain. ${ }^{50}$ The use of SMS in the HCP has been to achieve both higher spatial and temporal resolution fMRI. In the clinical domain, resting state fMRI has potential for use, in particular, for presurgical mapping. Importantly, this approach is less dependent upon patient compliance, requires less setup, and is easily acquired by MR technologists. A disadvantage, however, to resting state, when compared with task-based fMRI for clinical application, is scan time, which is inherently much longer for resting state fMRI as compared with task-based presurgical examinations (which can be performed in less than 10 minutes).

Heart. First-pass contrast-enhanced myocardial perfusion imaging is commonly used for detection of coronary artery disease. Constraints with current imaging technique include number of slices and (inplane) spatial resolution. In the absence of SMS, to sample more slices during every RR interval, each single-slice measurement would need to be shortened. Application of simultaneous multislice imaging enables a significant increase in number of acquired slices (typically $2 \times)$ and thus anatomic coverage, without any penalty or other change. In the multislice CAIPIRINHA approach, k-space undersampling is also used, to increase in-plane spatial resolution. ${ }^{51}$ The end result for first-pass perfusion imaging is the ability to obtain 6 slices for every heartbeat, with image quality comparable to a conventional 3-slice examination. Because image acquisition time is not shortened, only voxel size and the low g-factor noise amplification inherent with 

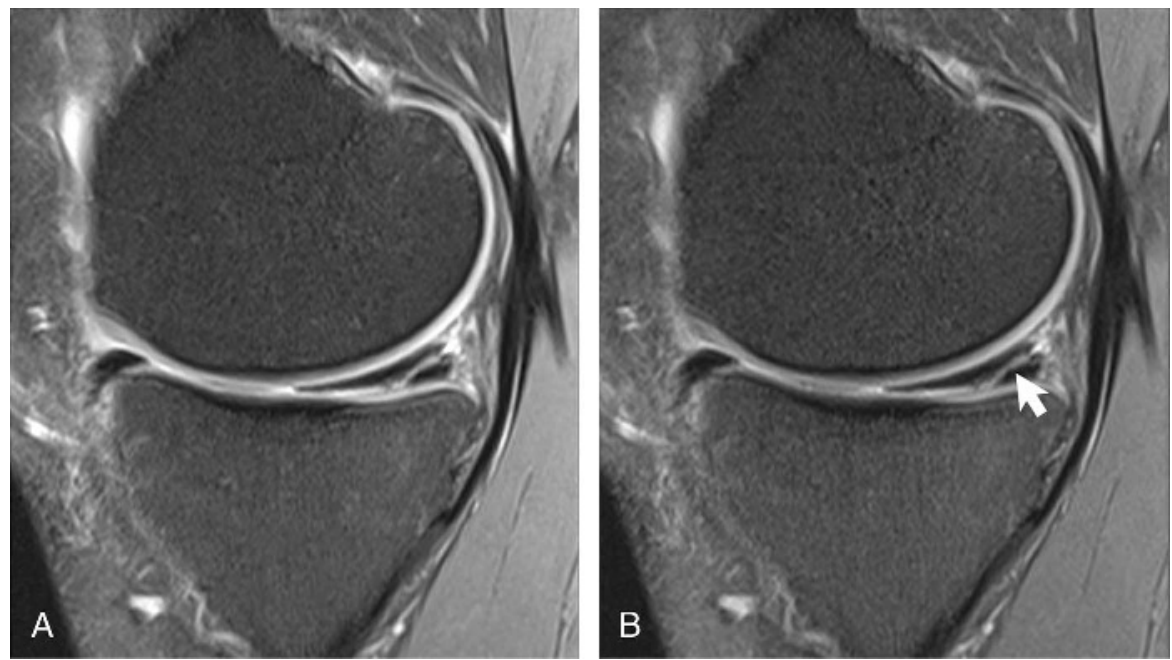

FIGURE 8. Sagittal proton density-weighted images of the knee demonstrating a horizontal tear (arrow) of the posterior horn of the medial meniscus on images (A) without and (B) with SMS (2× acceleration). Image quality and SNR are essentially equivalent, with a reduction in scan time from 6:36 to 3:27 min:s. This allows use of a high-resolution image protocol within an acceptable scan time, specifically an in-plane spatial resolution of $0.5 \times 0.4$ mm ${ }^{2}$ and a slice thickness of $2.5 \mathrm{~mm}$. Scans were acquired at $3 \mathrm{~T}$ using a 15-channel knee coil. Application of SMS allowed the use of a single concatenation (leading to the scan time reduction), as opposed to 2 concatenations with the non-SMS acquisition.

parallel image reconstruction affect SNR. Sufficient spatial resolution can be achieved in this fashion to clearly separate subendocardial from transmural perfusion defects, and to delineate small perfusion defects. Whole-heart coverage, which for an 8-mm slice thickness requires 12 sections, can be achieved using a temporal resolution of 1 measurement every 2 RR intervals.

Diffusion tensor imaging of the heart provides unique information regarding myocardial microstructure. ${ }^{52}$ Heart muscle is highly anisotropic, well suited for characterization by DTI. Myocardial fibers are arranged in sheets, with a continuum in helix angle (orientation relative to the short axis plane) change of about 180 degrees from their endocardial angle (+90 degrees) to that of the epicardium $\left(-90^{\circ}\right)$. A major limitation for the application of DTI in the heart, in addition to susceptibility, breathing, and heart motion, is scan time. Just 3 slices can require 20 minutes, and whole heart over 60 minutes. In initial evaluation, SMS techniques have been successfully used for tractography of the left ventricle with up to a threefold reduction in scan time.
Breast, Abdomen, and Pelvis. Diffusion-weighted imaging, which has become in the last decade a clinical standard in body imaging, is easily accelerated in this application with SMS. ${ }^{53}$ The utility of DWI in general for body imaging has been demonstrated in the breast, liver, pancreas, kidney, prostate, and uterus. Acquisition is typically during free-breathing, using single-shot SE echo-planar technique, with or without incorporation of a navigator. As in other areas of the body, with simultaneous excitation of multiple slices, the TR needed for the desired anatomic coverage can be reduced, leading to a reduction in scan time by the same factor (disregarding the small amount of time needed for the reference scan), without a significant SNR penalty (e.g., in breast imaging, Fig. 6). Although the technique could be used to achieve thinner sections (or higher in-plane resolution), given the effects of motion and the lower SNR in the body as compared with other areas of application of MR (such as the brain), the focus for use of SMS in the abdomen and pelvis has been to decrease scan time (Fig. 7).
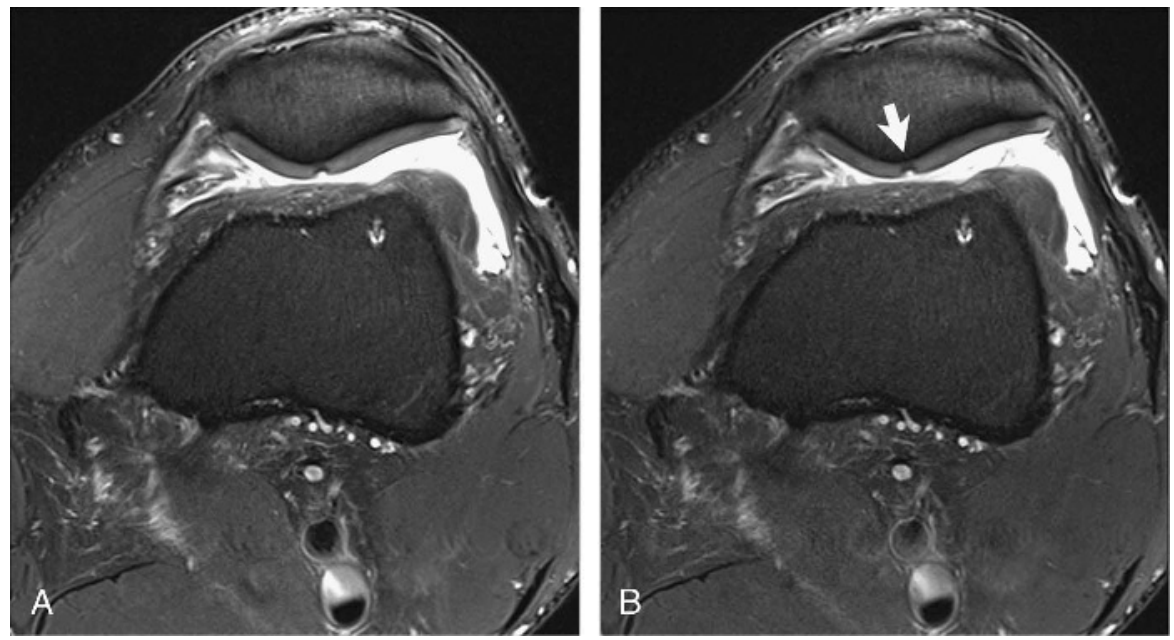

FIGURE 9. Axial TSE T2-weighted scans of the knee at 3 T depicting patellar cartilage damage (arrow), in a patient with prior medial collateral ligament and anterior cruciate ligament surgery, on images (A) without and (B) with SMS ( $2 \times$ acceleration). As in the previous figure, image quality and SNR are essentially equivalent, with a reduction in scan time from 7:14 to 3:48 min:s. The scan time with SMS is sufficiently short to make such a high spatial resolution acquisition (with a $0.4 \times 0.4 \mathrm{~mm}^{2}$ pixel) clinically feasible. 

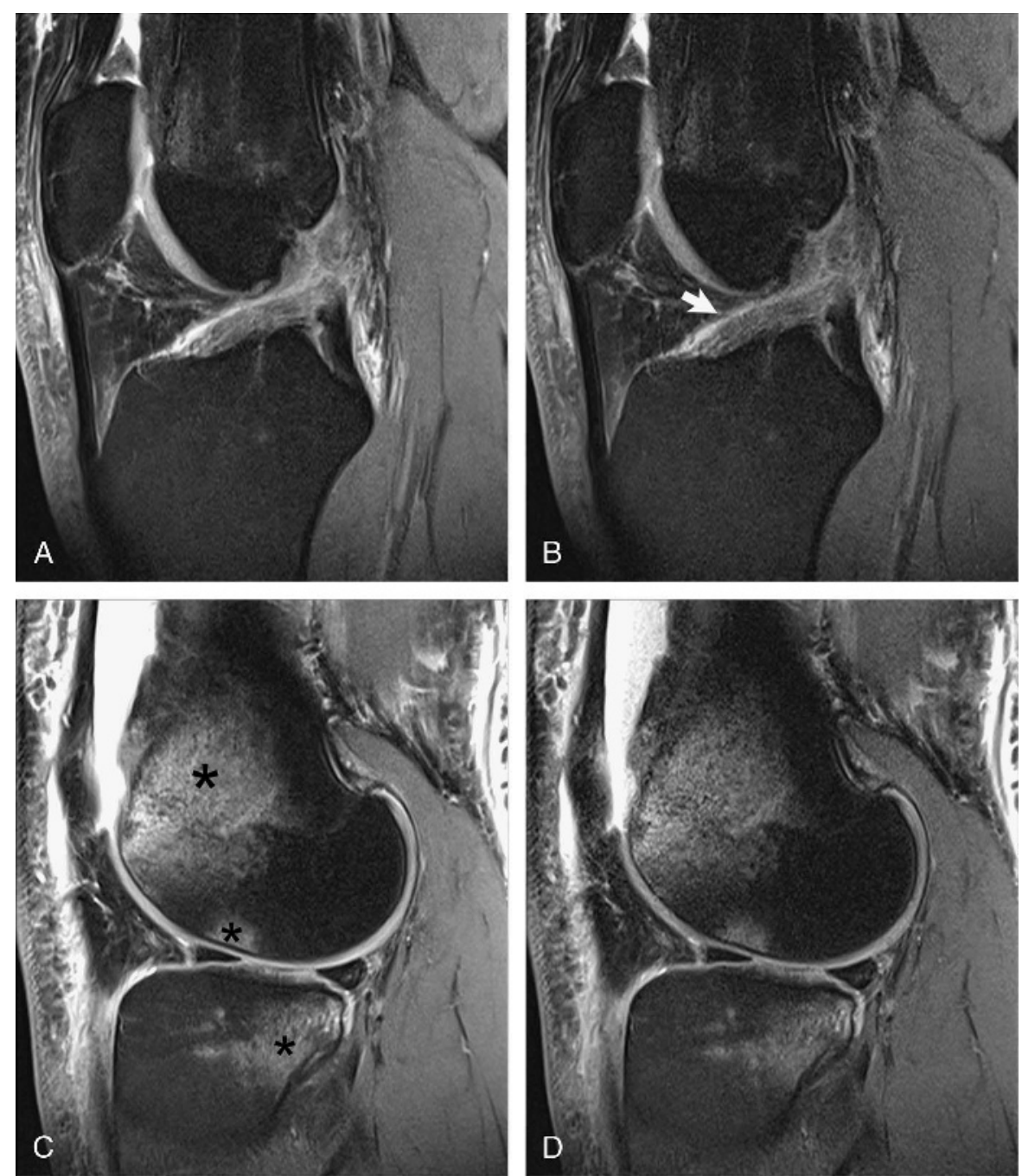

FIGURE 10. The use of SMS for abbreviated scan times in a trauma patient (motorcycle accident). A ruptured anterior cruciate ligament (arrow) is seen on ( $A$ and $B$ ) sagittal proton density-weighted images acquired at $3 \mathrm{~T}$, together with ( $\mathrm{C}$ and $\mathrm{D}$ ) bone marrow edema (asterisk) of the distal femur, lateral femoral condyle, and posterolateral tibial plateau. Depiction is essentially equivalent on the scans (A and C) without and (B and D) with SMS. The SMS scan, due to use of $2 \times$ acceleration, required only 1:34 min:s for acquisition, approximately half that for the non-SMS scan.

\section{Fast (Turbo) Spin Echo Imaging}

Clinical experience with FSE SMS is limited due to its very recent development and implementation. Anatomic applications will depend to some extent upon in which areas of the body imaging is currently limited by the available number of slices (in any single scan acquisition). Looking at the specific scan techniques that are used and the relative frequency of examinations by body part, it is evident that FSE SMS is likely to be heavily used in brain, axial spine, and musculoskeletal imaging (Figs. 8-10).

Thin section T2-weighted FSE images are acquired for every examination of the brain, with SMS thus likely having substantial impact in this area (Fig. 11). Due to potential heat deposition issues (in particular at $3 \mathrm{~T}$ ), implementation of low SAR VERSE pulses is important. In preliminary evaluation, these do not appear to have a substantial negative impact on image quality. Simultaneous multislice makes possible in less than 30 seconds whole-brain T2-weighted imaging with thin sections. Alternatively, thin sections with higher in-plane spatial resolution (e.g., $0.5 \times 0.5 \mathrm{~mm}^{2}$ ) can be acquired in scan times on the order of $2 \mathrm{mi}$ nutes, lessening the image degradation (in such higher resolution scans) due to inadvertent patient motion and enabling routine application in the clinic. This is of major benefit in patients with suspected brainstem pathology, both because these lesions can be minuscule, yet cause substantial neurological deficits, and due to the fact that some of these patients, often after a brainstem stroke, are agitated. Sedation in this instance cannot be used for the imaging examination, as it lowers the state of consciousness and thus limits the neurological examination options, potentially masking an aggravation of the symptoms.

Axial imaging of the spine represents another major area where FSE SMS is likely to see widespread use. Here, thin sections are required for clinical diagnosis, yet their use typically limits anatomic coverage. In this anatomic region, T2-weighted, T1-weighted, and T1-weighted postcontrast scans with fat saturation are all routinely acquired, and all can be applied with SMS

Similarly, there are many instances in musculoskeletal imaging in which the number of slices available for any given sequence is a limiting factor. In these anatomic regions (including specifically the knee and shoulder), the focus clinically is typically on T1-weighted and proton density-weighted (Figs. 8, 10) scans, both scan types being possible in early FSE implementations of SMS. As with other areas of the body, and with DWI SMS based scans, a decision tree emerges whether to use 

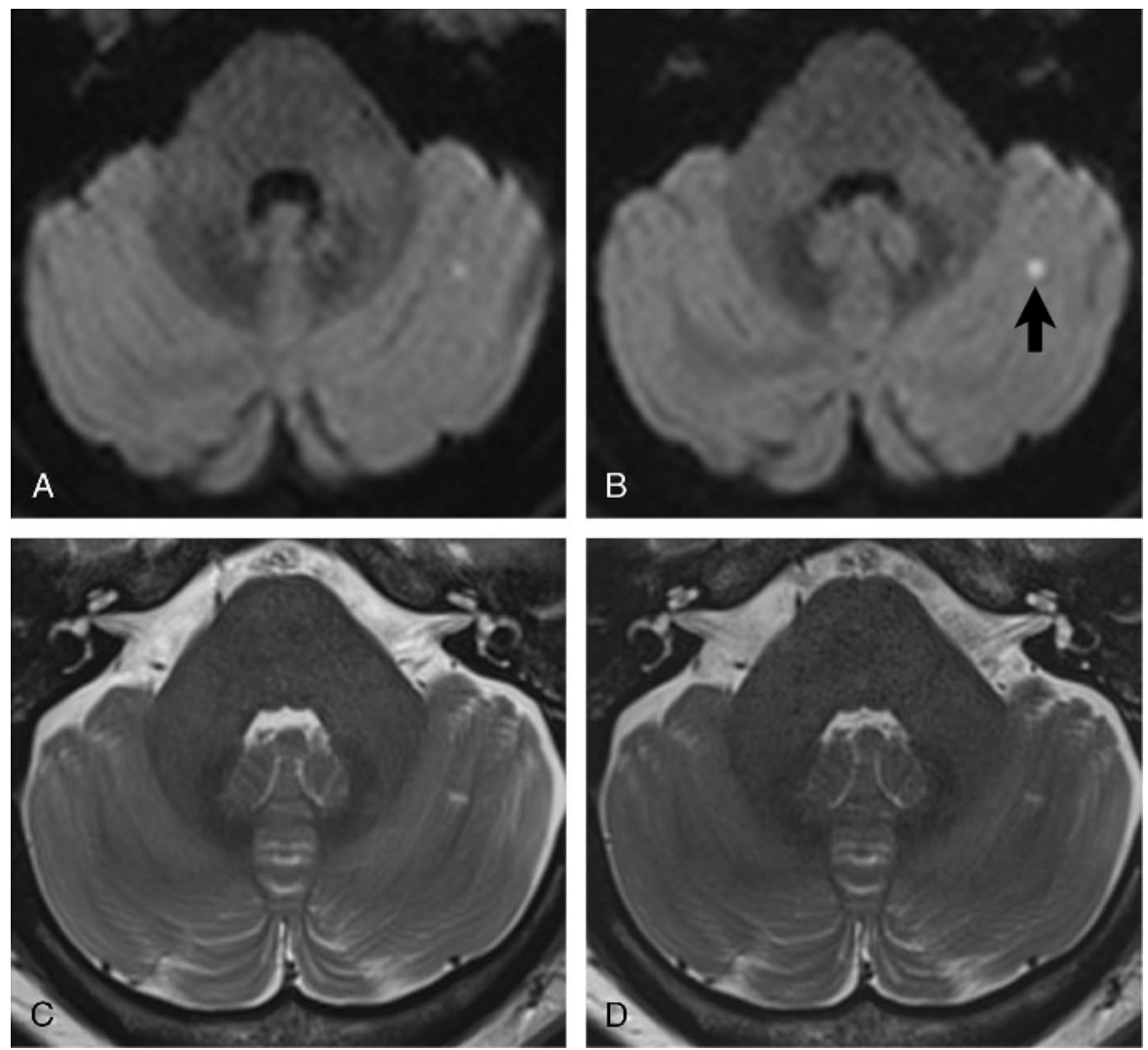

FIGURE 11. The use of SMS to decrease scan time for thin section imaging of the brain. A small pinpoint early subacute cerebellar infarct (arrow) is seen, on images acquired at $3 \mathrm{~T}$ using a 64-channel head and neck coil, in a 49-year-old female patient. For all scans, 2.5-mm-thick sections were acquired through the entire brain. Readout-segmented, diffusion-weighted $(b=1000)$ images are compared in the upper row, $(A)$ without and $(B)$ with SMS ( $2 \times$ acceleration). SMS was used to allow a decrease in TR from 12.75 to 6.38 seconds, with scan time thus decreasing from 5:46 to 3:26 min:s. In the lower row, T2-weighted scans (C) without and (D) with SMS ( $3 \times$ acceleration) are compared. In this instance, SMS was used to eliminate the need for concatenations ( 3 were required for the conventional scan, due to the number of slices), resulting in a decrease in scan time from 1:29 $\times 3$ (4:27) to 1:45 min:s. (Images courtesy of Marwan El-Koussy, Bern University Hospital).

SMS simply to decrease scan time, or to provide higher resolution images within a more acceptable scan time. Applicability is relatively independent of pathology, whether this involves tumors, or injuries to the menisci, tendons, ligaments and/or cartilage (Fig. 9).

\section{Future Impact}

Simultaneous multislice represents a further major technological advance in MR, with widespread applicability. Likely, it will come to be viewed as the major advance in the field during the current decade. Proper scanner hardware and software are critical to its implementation. Multielement array coils are required, with sufficient density of elements in the direction of slice encoding.

Discussed in the previous paragraphs are the many advantages inherent to the use of SMS. It is important to also note that, regardless of application, when used appropriately, there are few drawbacks to SMS. Although SAR constraints could be a limitation, with energy deposition proportional to the number of slices, to date, this has not adversely affected the implementation and use of EPI-based SMS techniques. Experience is much more limited with FSE SMS, and potential SAR limitations therein. It should also be kept in mind that it is very early in the development of SMS, and that substantial optimization of this technique is likely to occur over the decade to come, increasing further its utility and impact.

In a sense, SMS can be viewed as a further extension of very early imaging sequence developments in MR, which had a major impact on acquisition time, specifically multislice and FSE imaging. ${ }^{54}$ The initial implementation of SMS focuses mainly on using an acceleration factor of two, with the potential of the technique being much greater. However, in the clinical environment, a reduction in scan time by a factor of 2 is by itself a substantial, fundamental advance.

\section{Data Sparsity}

MR scans often can be long, making it challenging to obtain images with high resolution due to inadvertent patient motion. Certain organs can also be difficult to image, including the heart and the liver, due to physiologic motion, which includes breathing and cardiac contraction. Likewise rapid motion or changes in contrast can be difficult to image with MR, with the rapid change causing errors during data collection and degrading the final image.

In the last decade, image reconstruction techniques began to be explored that exploit data sparsity, to either reduce scan time and/or improve image quality. ${ }^{55}$ For example, one such technique that received substantial attention is compressed sensing. ${ }^{56}$ The fundamental basis for these techniques is that MR images contain redundant information. Thus it should be possible to undersample the data, speeding up image acquisition yet still capturing the essential information needed to maintain high image quality. Most of the current research is focused on conservative acceleration rates, given the early stage of the field and the occurrence of unpredictable artifacts. Long reconstruction times and the complexity of parameter optimization ${ }^{57}$ have also slowed development. 


\section{Theory}

Magnetic resonance imaging data are collected in a mathematical space called k-space. Data sparsity involves collecting less than the complete set of data, specifically undersampling k-space. Such undersampling can, however, lead to artifacts. A well-known example is sampling every other line of $\mathrm{k}$-space, which results in fold over artifacts (image wrap). ${ }^{13}$ Sparse reconstruction techniques depend upon the concepts of image sparsity, aliasing artifacts, and specialized reconstruction to recover the unaliased, sparse image (Fig. 12). Images may have data sparsity spatially or temporally. Thus MRI techniques that observe a dynamic process, such as cardiac motion or contrast enhancement are good areas potentially for the application of sparse reconstruction. Regarding aliasing artifacts, a requirement for sparse reconstruction techniques is that the true signal must be distinguishable from the benign noise or aliasing artifact that arises from undersampling. Sparse reconstruction techniques rely upon the use of a specialized data sampling pattern that results in nonregular, noise-like aliasing artifacts (which can be achieved, e.g., by randomly undersampling k-space), with the actual image readily distinguishable from these. The selection of an appropriate trajectory through $\mathrm{k}$-space for data collection is extremely important, with 1 requirement being that the path must be smooth. Thus, with 2D imaging, in the place of random undersampling, other trajectories are used, for example, radial or spiral paths. The information used to recover unaliased images, the third major tenant of sparse reconstruction techniques, varies greatly depending on the method, with currently 4 major groups of techniques, which are subsequently described. For greater detail, the reader is referred to an excellent recent review of the methods, applications, and challenges to clinical adoption of sparse reconstruction techniques in MR. ${ }^{13}$

\section{Reconstruction Techniques}

Separable methods are based upon the concept that spatial information can be separated from temporal information in a dynamic image series. Low-resolution images capturing temporal dynamics are used together with a static high spatial resolution image to generate a dynamic series with high spatial resolution. These methods rely upon the assumption that the change from frame to frame is only in a few image pixels, and that the change is slow and smooth. HYPRFlow is one such technique, used for MR angiography (MRA), and assumes that the signal intensity changes are restricted to blood vessels. ${ }^{58}$ Although such methods have been most frequently applied to contrast-enhanced MRA, it may also be possible to accelerate scans used to evaluate tissue perfusion, for example, the myocardium or the liver.

k-t methods, like separable methods, take advantage of sparsity in dynamic acquisitions, but use both spatial and temporal sparsity. ${ }^{59}$ Many variants of this approach exist, but all depend upon the principle that for many dynamic images (e.g., cardiac imaging) only certain parts of the image contain motion with the rest static.

The basic concept of compressed sensing is that if MR data are acquired in such a fashion that undersampling artifacts appear as noise,

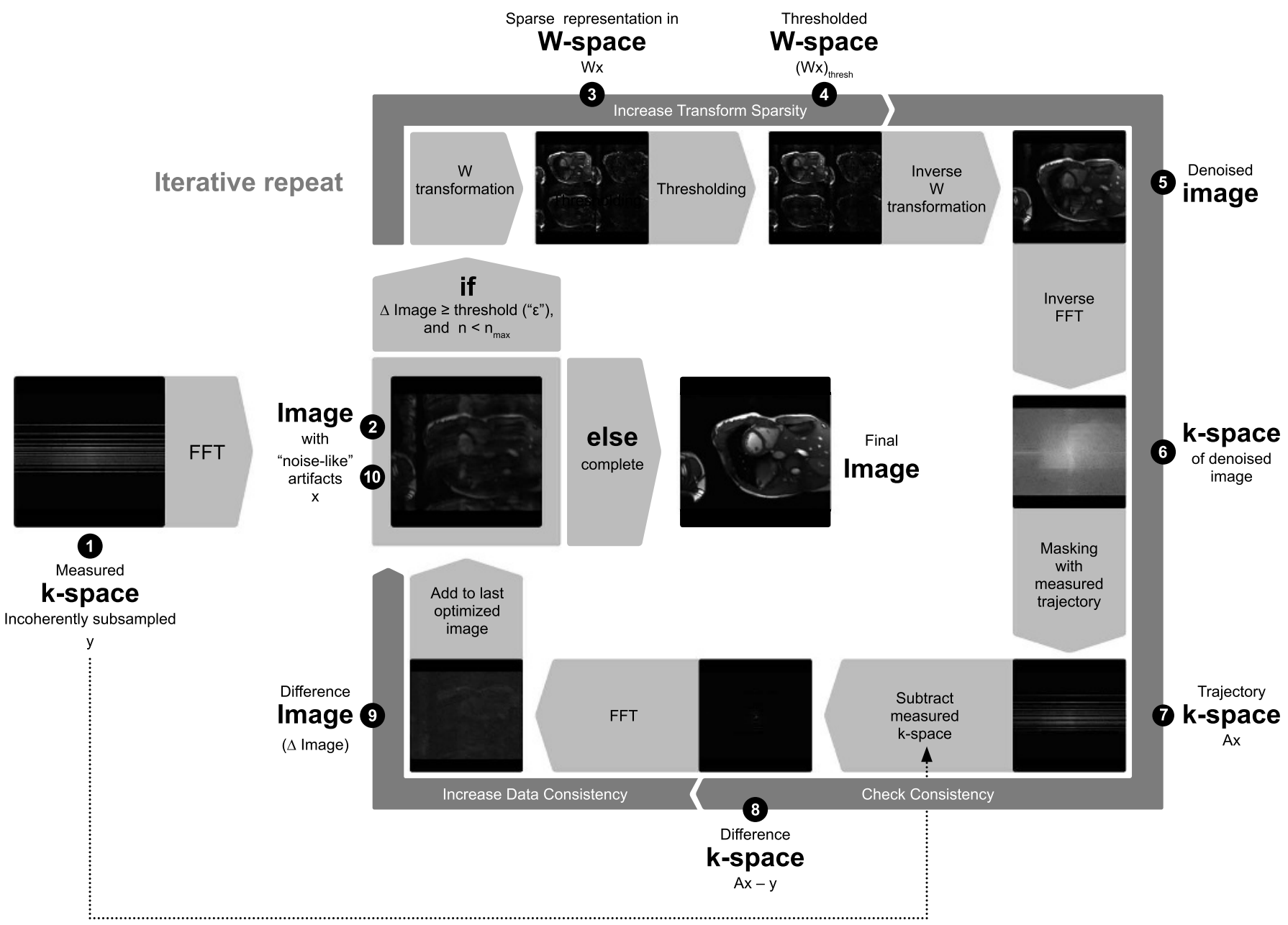

FIGURE 12. Diagrammatic representation of data acquisition and reconstruction using the concept of data sparsity, with undersampling of k-space and subsequent iterative thresholding and denoising, leading eventually to the final reconstructed image. 

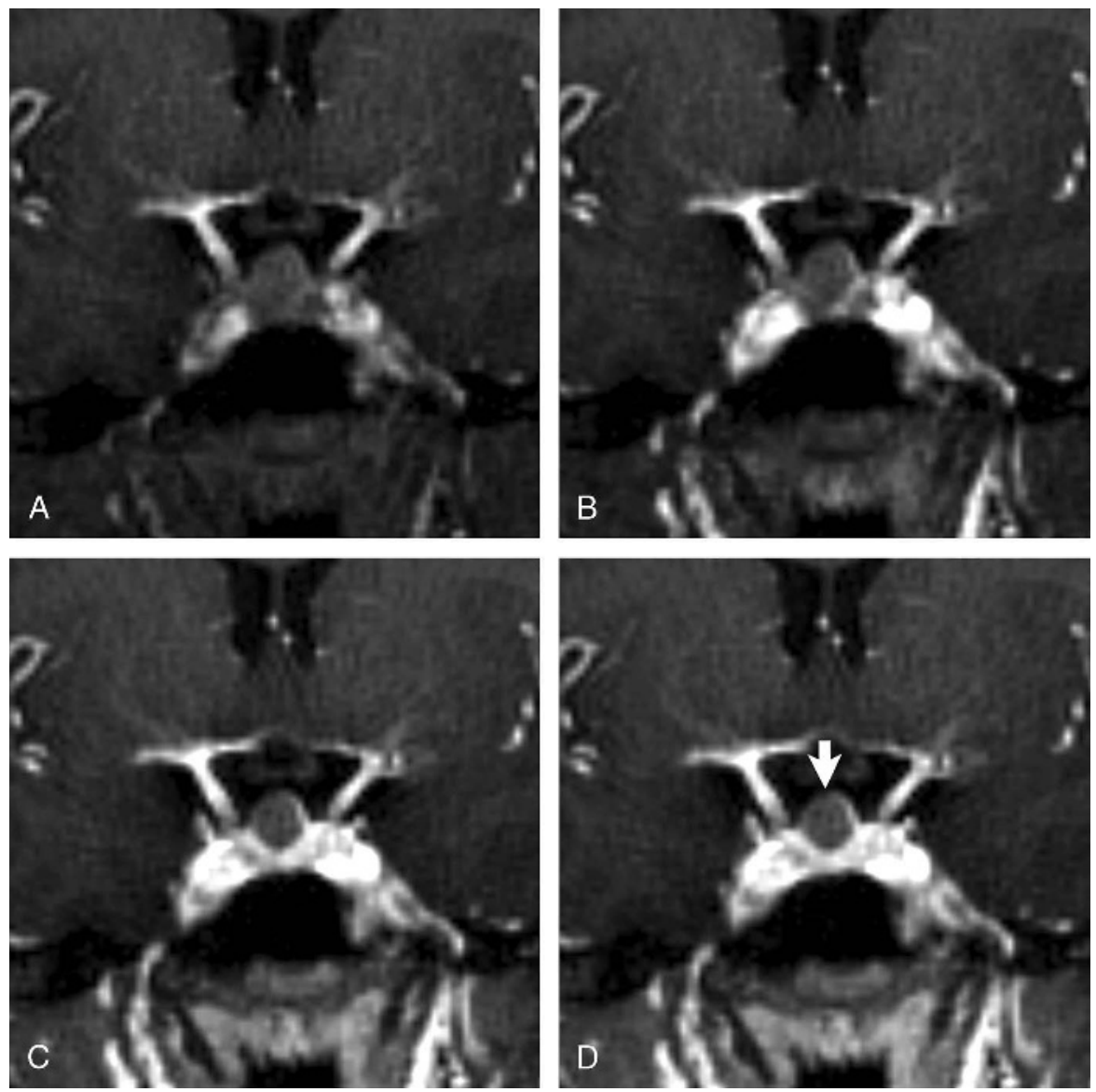

FIGURE 13. Dynamic contrast-enhanced high-resolution imaging of the pituitary using sparse sampling at $3 \mathrm{~T}$. A GRASP sequence was used, which used both compressed sensing and parallel imaging. The voxel size was $1 \times 1 \times 1 \mathrm{~mm}^{3}$ and the temporal resolution 4 seconds. A cystic, nonenhancing, nonhormonally active, lesion (arrow) of the pituitary is well depicted on scans acquired at (A) 46 seconds, (B) 54 seconds, (C) 2:26 min:s, and (D) 2:54 min:s (selected images from the rapid continuous acquisition are illustrated). By 2 minutes, enhancement of the normal pituitary is evident caudal to the lesion. (Images courtesy of Andrea Bink, University Hospital of Basel).

and the data are sparse, then the full image can be recovered with an appropriate reconstruction technique. The mathematical calculation involves seeking, in an iterative fashion, a final image that is sparse (or sparse in the transform domain) and consistent with the acquired data. A regularization parameter controls the relative importance of these 2 terms, and can be chosen to lead to a solution closer to or deviating more away from the collected data, the latter relying more on the sparsity term. Unlike the previous 2 methods described, compressed sensing can be used to accelerate static (nondynamic) imaging. Compressed sensing can also be used in dynamic imaging. Motion, including respiration and cardiac contraction, can negatively affect the efficacy of compressed sensing reconstruction, and thus the approach has been combined in some applications with navigator and image registration techniques. Golden-angle radial sparse parallel is one of many compressed sensing techniques, with radial acquisition of continuously acquired dynamic 3D information. ${ }^{26}$

The fourth major category of sparse reconstruction techniques is the model-based methods. In this approach, the fundamental assumption is that the final image, or dynamic images, can be described by a set of parameters that is much smaller than the number of pixels. One way of using a model to perform a sparse reconstruction is to use a dictionary-based approach, matching the pixel time course with a dictionary of possible courses. Magnetic resonance fingerprinting (providing quantitative maps of T1, T2, proton density, and diffusion) is one such dictionary-based approach. ${ }^{60}$ Any physical property can be treated in this way, if a well-defined mathematical relationship is known between the property and the signal time course. This includes, in addition to that already mentioned, fat fraction and susceptibility mapping. Another approach, within the category of data-driven undersampling methods, is to use at least 1 fully sampled scan as a training set, with such use improving subsequent reconstruction quality. ${ }^{61}$

\section{Clinical Studies/Applications}

\section{Neuroimaging}

There are many techniques in MR neuroimaging, which today are impractical due to very long scan times, which could benefit if sparse reconstruction techniques prove viable. These include quantitative relaxometry techniques such as MR fingerprinting, and advanced/ improved applications of fMRI and DTI (fiber tracking).

Techniques using radial undersampling and sparse reconstruction are already available for time-resolved contrast-enhanced MRA, a major clinical application. HYPRFlow is one such approach, allowing high spatial $(0.7 \mathrm{~mm}$ isotropic) and temporal $(0.8$ second $)$ resolution with whole-brain coverage. Its value has been shown for characterizing arteriovenous malformations, with excellent concordance to digital subtraction angiography in determination of Spetzler-Martin grade as well as assessment of venous ectasia and deep venous drainage. ${ }^{62}$ Arterial image quality was shown to be equivalent to 3D time-of-flight (TOF) MRA, with excellent arterial and venous separation. The utility of this 
technique has also been demonstrated in the delineation of dural arteriovenous fistulas (dAVFs), specifically their arterial and venous components as well as arteriovenous shunting, allowing correct classification using the Cognard system. ${ }^{58}$

The combination of radial imaging with compressed sensing implemented in GRASP has also provided a real-world solution to dynamic imaging of the pituitary, an area where high spatial and temporal resolutions are desired. These 2 requirements both demand SNR, and thus scans have traditionally emphasized 1 of the 2 . Scans can now be acquired during bolus intravenous contrast administration, by the use of data sparsity techniques, with both high spatial and temporal resolution, permitting identification of very small lesions within the normal-enhancing pituitary and differentiation between small cysts and microadenomas (Fig. 13).

\section{Cardiac Imaging}

Despite the excellent image quality achieved with cardiac MRI today, well-displaying myocardial function, conventional imaging leads to long acquisition times and depends on patient compliance to achieve high spatial and temporal resolution. In current clinical practice, ventricular function assessment is part of almost every cardiac examination, being an important predictor of patient outcome. CINE images are well suited for compressed sensing because sparsity along both the spatial and temporal domains can be exploited for incoherent subsampling. Real-time imaging using this approach has been demonstrated, in clinical studies, with similar temporal and spatial resolution as in conventional segmented imaging. ${ }^{63,64}$ Real-time imaging has not only the potential to significantly speed up the examination by reducing the scan time for left or right ventricular assessment to 1 breath-hold, but also facilitates imaging in free-breathing and is robust against arrhythmia.

In addition, high-resolution 3D MRI is well suited for compressed sensing, for example, in coronary MRA. Thanks to the denoising capabilities of compressed sensing, imaging can either be significantly accelerated or performed with higher resolution while maintaining image quality. ${ }^{65}$ First-pass perfusion imaging and late gadolinium enhancement imaging both also can potentially benefit by the application of sparse reconstruction, enabling improved image quality, higher spatial resolution, and/or in the case of first-pass imaging higher temporal resolution.

\section{Lung Imaging}

Areas of application for sparse reconstruction techniques in lung imaging include high-resolution structural assessment of the lungs, imaging of small tumors, and dynamic free-breathing evaluation. ${ }^{66}$ Whether high temporal resolution acquisitions, with or without sparse reconstruction, will make pulmonary MRI a feasible alternative to CT is, however, yet to be determined.

\section{Abdominal and Pelvic Imaging}

Early publications focused on the application of sparse reconstruction for improving abdominal imaging in children, given the difficulty in obtaining the breath-hold scans, and inadvertent patient motion, both intrinsic to the age of the patient. ${ }^{67}$ An important advance would be if anesthesia can be avoided by application of such techniques.

In the general patient population, application of sparse reconstruction may provide more robust abdominal perfusion imaging, with large volume coverage and high spatial resolution. Free-breathing radial acquisition schemes incorporating compressed sensing, such as GRASP, have recently become available clinically and enable in a single examination both morphologic assessment and the temporal resolution necessary for pharmacokinetic modeling. ${ }^{68}$ For example, in the publication cited, reconstructions were performed with both 55 and 13 consecutive spokes, achieving temporal resolutions of 10 seconds (for clinical evaluation) and 2.2 to 2.4 seconds (for pharmacokinetic analysis), respectively, after intravenous bolus disodium gadoxetate injection. The initial research with this technique was performed for liver imaging, with results recently extended to the kidney. ${ }^{69}$

Oversampling of the center of k-space in radial imaging can also be used for self-navigation, and thus exploited to minimize the blurring due to respiratory motion. In 1 current implementation, a compressed sensing algorithm exploiting sparsity is used for both the contrast enhancement and respiratory/cardiac dimensions, a technique called XD-GRASP. ${ }^{70}$ This has been used for multiphase dynamic contrastenhanced liver imaging and compared (using the end expiratory phase reconstruction) to GRASP and conventional breath-hold Cartesiansampled data (VIBE). XD-GRASP proved equal to superior in terms of overall image quality (in most instances with statistical significance) to both comparators (Fig. 14) in comparisons of early arterial, late arterial, and portal venous images. ${ }^{71}$
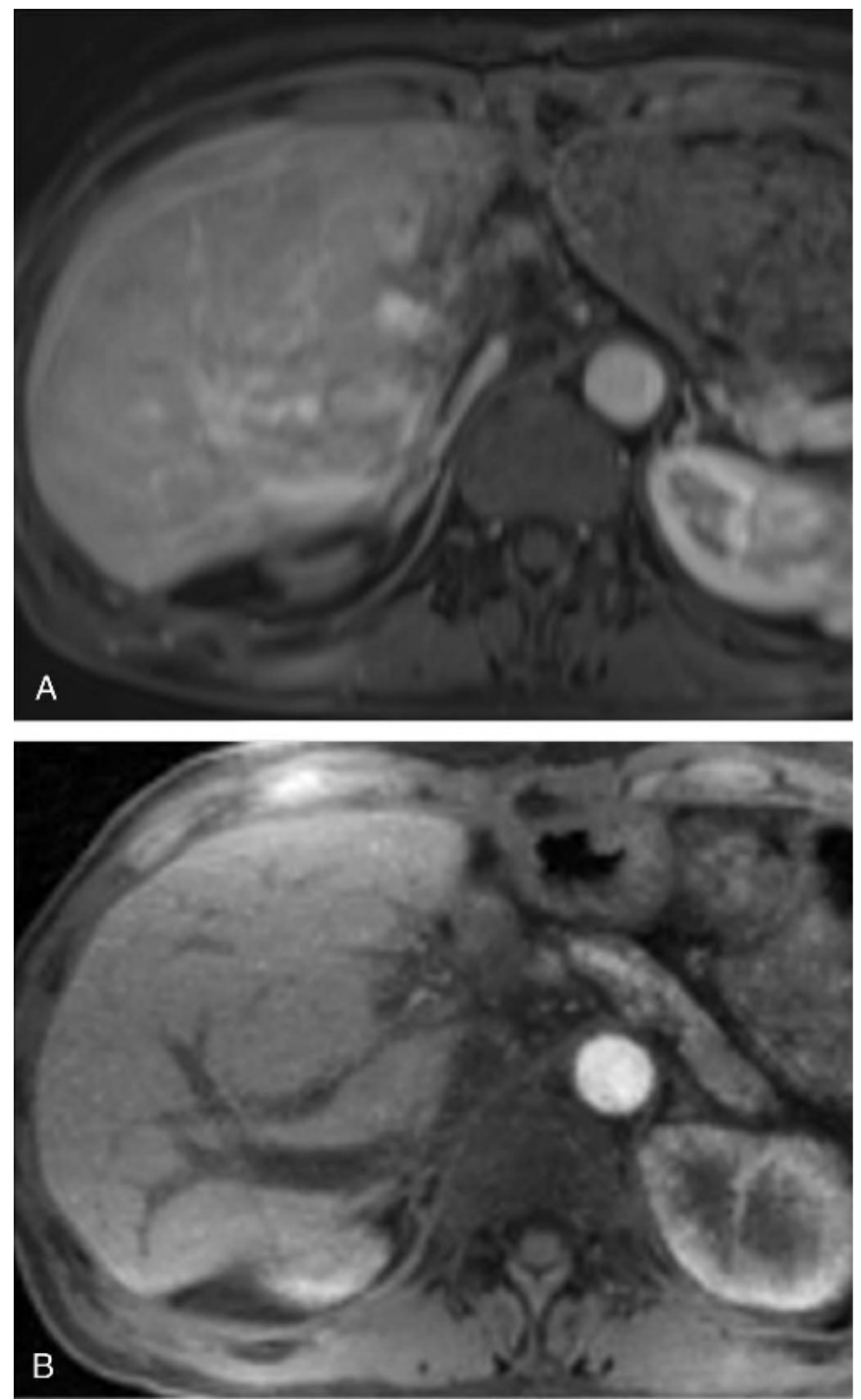

FIGURE 14. Comparison of (A) breath-hold Cartesian and (B) free-breathing XD (extradimensional) GRASP liver images in a 40-year-old female patient at $1.5 \mathrm{~T}$, acquired during the early arterial phase after gadoxetic acid administration. The lack of a good breath-hold degrades substantially the conventional image, with the free-breathing radial acquisition demonstrating markedly improved and, specifically, diagnostic quality images. (Images courtesy of Hersh Chandarana, NYU Langone Medical Center, New York and Invest Radiol 2015;50(11):749-756). 
As in other areas of the body, data sparsity can be used to shorten scan times, with particular applicability in the upper abdomen, due to the potential to acquire high-resolution 3D images with a large FOV within a reasonable breath-hold period. In a recent study, a breath-hold accelerated SPACE acquisition with only $5 \%$ sampling of $\mathrm{k}$-space was compared with a more conventional respiratory-triggered acquisition for the assessment of the biliary and pancreatic ducts. ${ }^{72}$ The sparse SPACE acquisition showed similar or superior image quality for assessment of the ducts despite the 17 -fold shorter scan time.

\section{Musculoskeletal Imaging}

A major clinical application of sparse reconstruction is musculoskeletal imaging in the presence of metal, for example, with joint prostheses (Fig. 15). Early work has applied compressed sensing acceleration to slice encoding for metal artifact correction (SEMAC) with evaluation for imaging of cobalt chromium knee implants. Scan time was reduced from 11 minutes to less than 5 minutes, with image quality assessments superior or equivalent to the SEMAC scan without compressed sensing for all but 1 of 23 compared parameters. ${ }^{73}$ Another possible area of application is simply that of accelerating isotropic high-resolution 3D imaging of individual joints, with preliminary experience in knee imaging. ${ }^{74} \mathrm{Al}-$ though many other areas hold promise for application, such as cartilage imaging and, at $7 \mathrm{~T}$, sodium and ${ }^{31} \mathrm{P}$ spectroscopic imaging, these examinations remain in the research realm.

\section{Vascular and Flow Imaging}

Magnetic resonance angiography is a natural application for data sparsity. Magnetic resonance angiograms are sparse in the image domain, and for contrast-enhanced examinations also in the time domain. Potential major applications include imaging of the cerebral arterial vasculature with TOF MRA ${ }^{75}$ and evaluation of the carotid arteries, coronary arteries, and pulmonary vasculature. In contrast-enhanced MRA, the concept of data sparsity can be used to reduce acquisition time, enabling more rapid temporal imaging with separate visualization of the arterial and venous phases. This has widespread applicability, in particular for vessel stenoses or occlusions and for arteriovenous malformations and dAVFs (as discussed in the neuroimaging section). Sparse reconstruction techniques also hold particular promise in areas where respiratory and/or cardiac motion cause substantial problems.
Sparse reconstruction has been explored in a preliminary fashion for improved 4-dimensional flow imaging, with applications including valvular insufficiency and congenital heart disease. High-resolution carotid imaging may also substantially benefit, with higher acceleration factors allowing improved vessel delineation and flow quantification, within a clinically acceptable imaging time.

\section{Challenges}

Although sparse reconstruction techniques show promise, the field is early in its development with selection of specific technique and optimization therein still largely unclear. Despite these challenges, in several specific areas clinical applicability has been demonstrated, providing substantial improvements in diagnostic efficacy. This includes, for example, applications in TOF MRA, CE-MRA, cardiac CINE, GRASP (for liver imaging), tissue perfusion, and metal artifact reduction.

One difficulty is the plethora of techniques being evaluated, and the numerous areas that they might be applied in. Another is the difficulty of clinical evaluation of the techniques, which in the end requires within patient comparisons against current clinical standard techniques. In studies depending upon contrast injection, this would also require 2 separate patient examinations. An additional major challenge is that each method strongly depends upon the parameters used in the reconstruction, and the optimization thereof. Improper selection leads to images in which essential features can be lost. To complicate things further, optimal values can be substantially different between methods, and values apply only for the specific application in which they have been used and optimized. Performance of methods can also be highly dependent upon how the data are sampled and the degree of undersampling. The algorithms used for sparse reconstruction methods are complex, with proper implementation challenging. Thus assessing different approaches is difficult, although there have been attempts to so compare the results of different research groups and their techniques for image reconstruction, with some success.

Sparse reconstruction techniques are in general computationally intensive, necessitating optimization for clinical implementation. As with other innovations over the history of MR, the computational demands will likely not represent a substantial hindrance to the implementation of sparse reconstruction techniques, given the continued advance
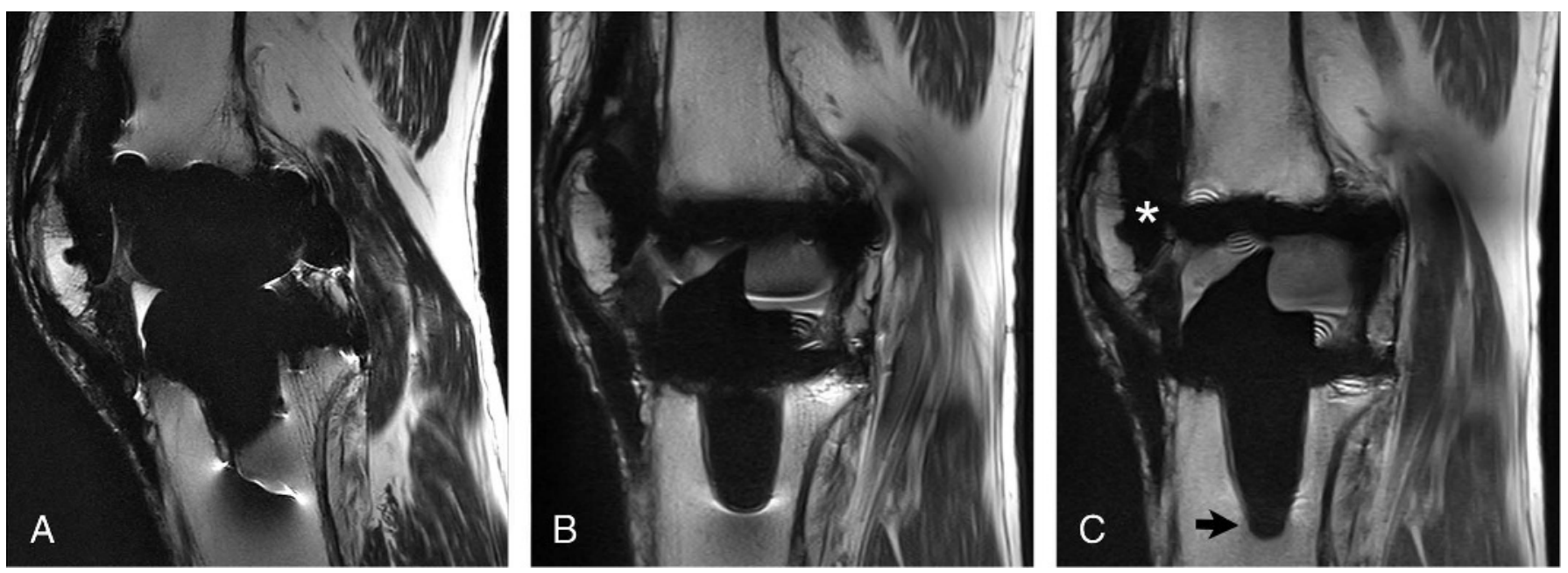

FIGURE 15. Sparse sampling allowing an increased number of SEMAC steps in a reasonable scan time for improved metal artifact reduction in orthopedic implant imaging. This 61 -year-old man had a total knee arthroplasty and subsequently developed arthrofibrosis (asterisk). All images were acquired at 1.5 T. Sagittal TSE images are compared with (A) high bandwidth, (B) conventional SEMAC (12 steps), and (C) SEMAC with sparse sampling (15 steps). Scan times were 3:40, 4:50, and 4:22 min:s, respectively. Artifacts from the femoral component of the arthroplasty are almost completely absent in panel C, with 15 SEMAC steps sufficient for complete visualization of the tibial peg (arrow) and 12 steps not. (Images courtesy of Reto Sutter, Balgrist University Hospital, Zurich). 
in computational techniques, and hardware, and lower costs. Along this line, it should be kept in mind that in the early 1980 s, a state-of-the-art dedicated array processor used for MR systems required 10 seconds for reconstruction of a single $128 \times 128$ matrix image (something that requires only milliseconds today). One further challenge for clinical implementation of data sparsity reconstruction techniques involves potential artifacts in the final images. These may be to some degree unpredictable and will no doubt be unfamiliar to the radiologists involved in early image interpretation.

In the near term future, there are a number of common clinical examinations where sparse reconstruction techniques will have immediate application, and where this represents a major advance over current imaging approaches. Discussed in order in terms of applicable examinations in day-to-day clinical practice, these include brain, abdominal, and musculoskeletal applications. For the brain, most TOF and CE-MRA examinations can benefit, with these 2 examinations being frequently used clinically (and for TOF in many centers for every patient). In terms of the application of CE-MRA, although studies have shown utility in arteriovenous malformations and dAVFs, the potential use is far greater, including all mass lesions and brain ischemia. In a smaller but critical patient population, imaging of the pituitary will benefit in all instances by the use of sparse reconstruction techniques, enabling dynamic examinations with both high spatial and temporal resolution after intravenous contrast administration, improving lesion identification, characterization, and differentiation from the normal gland. In abdominal MR, non-breath-hold multiphase dynamic contrast-enhanced examinations using sparse reconstruction techniques are posed to replace the conventional breath-hold arterial, venous, and portal examinations, improving in a large patient population image quality and lesion depiction, allowing in addition pharmacokinetic lesion analysis. Magnetic resonance cholangiopancreatography examinations will also benefit, with likely replacement of current techniques by a single, breath-hold, accelerated examination. In musculoskeletal imaging, imaging of prostheses will also immediately benefit, by implementation of advanced metal reduction strategies, with application, for example, using a higher number of SEMAC encoding steps for improved depiction of structures adjacent to the prosthesis yet with the scan acquired in under 5 minutes.

\section{CONCLUSIONS}

Sparse imaging and SMS represent the 2 fundamental advances in the current decade with major current and future positive impact upon MR clinical use and diagnosis therein. These build upon the foundation laid in the past 20 years by parallel imaging, GRAPPA, VIBE, TWIST, and radial imaging. These 2 imaging approaches apply both to specialty examinations in MR, such as metal artifact reduction, and more importantly to general clinical utilization, for example, imaging of the brain. "Speed" is a critical issue for MR, with the definition of speed including not just how fast the scan itself can be but also extending to the robustness of the technique and the speed of data sampling. Regardless of anatomic area and desired specific tissue contrast, sparse imaging and SMS can be applied, and will fundamentally impact the clinical utilization of MR, with further developments therein extending over at least the next decade.

\section{REFERENCES}

1. Runge VM, Price AC, Kirshner HS, et al. Magnetic resonance imaging of multiple sclerosis: a study of pulse-technique efficacy. AJR Am J Roentgenol. 1984; 143:1015-1026.

2. Runge VM, Clanton JA, Partain CL, et al. Respiratory gating in magnetic resonance imaging at 0.5 Tesla. Radiology. 1984;151:521-523.

3. Sodickson DK, Manning WJ. Simultaneous acquisition of spatial harmonics (SMASH): fast imaging with radiofrequency coil arrays. Magn Reson Med. 1997;38:591-603.

4. Griswold MA, Jakob PM, Heidemann RM, et al. Generalized autocalibrating partially parallel acquisitions (GRAPPA). Magn Reson Med. 2002;47:1202-1210.
5. Breuer FA, Blaimer M, Mueller MF, et al. Controlled aliasing in volumetric parallel imaging (2D CAIPIRINHA). Magn Reson Med. 2006;55:549-556.

6. Mistretta CA, Grist TM. X-ray digital subtraction angiography to magnetic resonance-digital subtraction angiography using three-dimensional TRICKS. Historical perspective and computer simulations: a review. Invest Radiol. 1998;33 496-505.

7. Turski PA, Korosec FR, Carroll TJ, et al. Contrast-enhanced magnetic resonance angiography of the carotid bifurcation using the time-resolved imaging of contrast kinetics (TRICKS) technique. Top Magn Reson Imaging. 2001;12:175-181.

8. Lim RP, Shapiro M, Wang EY, et al. 3D time-resolved MR angiography (MRA) of the carotid arteries with time-resolved imaging with stochastic trajectories: comparison with 3D contrast-enhanced Bolus-Chase MRA and 3D time-of-flight MRA. AJNR Am J Neuroradiol. 2008;29:1847-1854.

9. Michaely HJ, Morelli JN, Budjan J, et al. CAIPIRINHA-Dixon-TWIST (CDT)volume-interpolated breath-hold examination (VIBE): a new technique for fast time-resolved dynamic 3-dimensional imaging of the abdomen with high spatial resolution. Invest Radiol. 2013;48:590-597.

10. Chandarana H, Block TK, Rosenkrantz AB, et al. Free-breathing radial 3D fatsuppressed T1-weighted gradient echo sequence: a viable alternative for contrast-enhanced liver imaging in patients unable to suspend respiration. Invest Radiol. 2011;46:648-653.

11. Setsompop K, Gagoski BA, Polimeni JR, et al. Blipped-controlled aliasing in parallel imaging for simultaneous multislice echo planar imaging with reduced g-factor penalty. Magn Reson Med. 2012;67:1210-1224.

12. Barth M, Breuer F, Koopmans PJ, et al. Simultaneous multislice (SMS) imaging techniques. Magn Reson Med. 2016;75:63-81.

13. Yang AC, Kretzler M, Sudarski S, et al. Sparse reconstruction techniques in magnetic resonance imaging: methods, applications, and challenges to clinical adoption. Invest Radiol. 2016;51:349-364.

14. Roemer PB, Edelstein WA, Hayes CE, et al. The NMR phased array. Magn Reson Med. 1990;16:192-225.

15. Pruessmann KP, Weiger M, Scheidegger MB, et al. SENSE: sensitivity encoding for fast MRI. Magn Reson Med. 1999;42:952-962.

16. Jakob PM, Griswold MA, Edelman RR, et al. AUTO-SMASH: a self-calibrating technique for SMASH imaging. SiMultaneous Acquisition of Spatial Harmonics. MAGMA. 1998;7:42-54.

17. Riffel P, Attenberger UI, Kannengiesser S, et al. Highly accelerated T1-weighted abdominal imaging using 2-dimensional controlled aliasing in parallel imaging results in higher acceleration: a comparison with generalized autocalibrating partially parallel acquisitions parallel imaging. Invest Radiol. 2013;48:554-561.

18. Park YS, Lee CH, Kim IS, et al. Usefulness of controlled aliasing in parallel imaging results in higher acceleration in gadoxetic acid-enhanced liver magnetic resonance imaging to clarify the hepatic arterial phase. Invest Radiol. 2014;49: $183-188$.

19. Kazmierczak PM, Theisen D, Thierfelder KM, et al. Improved detection of hypervascular liver lesions with CAIPIRINHA-Dixon-TWIST-volume-interpolated breath-hold examination. Invest Radiol. 2015;50:153-160.

20. Othman AE, Martirosian P, Schraml C, et al. Feasibility of CAIPIRINHA-DixonTWIST-VIBE for dynamic contrast-enhanced MRI of the prostate. Eur J Radiol. 2015;84:2110-2116.

21. Othman AE, Falkner F, Martirosian P, et al. Optimized fast dynamic contrastenhanced magnetic resonance imaging of the prostate: effect of sampling duration on pharmacokinetic parameters. Invest Radiol. 2016;51:106-112.

22. Chandarana H, Block KT, Winfeld MJ, et al. Free-breathing contrast-enhanced T1-weighted gradient-echo imaging with radial k-space sampling for paediatric abdominopelvic MRI. Eur Radiol. 2014;24:320-326.

23. Bamrungchart S, Tantaway EM, Midia EC, et al. Free breathing three-dimensional gradient echo-sequence with radial data sampling (radial 3D-GRE) examination of the pancreas: Comparison with standard 3D-GRE volumetric interpolated breathhold examination (VIBE). J Magn Reson Imaging. 2013;38:1572-1577.

24. Bangiyev L, Raz E, Block TK, et al. Evaluation of the orbit using contrastenhanced radial 3D fat-suppressed T1 weighted gradient echo (Radial-VIBE) sequence. Br J Radiol. 2015;88:20140863.

25. Wu X, Raz E, Block TK, et al. Contrast-enhanced radial 3D fat-suppressed T1 weighted gradient-recalled echo sequence versus conventional fat-suppressed contrast-enhanced T1-weighted studies of the head and neck. AJR Am J Roentgenol. 2014;203:883-889.

26. Chandarana H, Feng L, Block TK, et al. Free-breathing contrast-enhanced multiphase MRI of the liver using a combination of compressed sensing, parallel imaging, and golden-angle radial sampling. Invest Radiol. 2013;48:10-16.

27. Edelstein WA, Hutchison JM, Johnson G, et al. Spin warp NMR imaging and applications to human whole-body imaging. Phys Med Biol. 1980;25:751-756.

28. Alfidi RJ, Haaga JR, El-Yousef SJ, et al. Preliminary experimental results in humans and animals with a superconducting, whole-body, nuclear magnetic resonance scanner. Radiology. 1982;143:175-181. 
29. Crooks LE, Ortendahl DA, Kaufman L, et al. Clinical efficiency of nuclear magnetic resonance imaging. Radiology. 1983;146:123-128.

30. Larkman DJ, Hajnal JV, Herlihy AH, et al. Use of multicoil arrays for separation of signal from multiple slices simultaneously excited. J Magn Reson Imaging. 2001; 13:313-317.

31. Souza SP, Szumowski J, Dumoulin CL, et al. SIMA: simultaneous multislice acquisition of MR images by Hadamard-encoded excitation. J Comput Assist Tomogr. 1988;12:1026-1030.

32. Feinberg DA, Moeller S, Smith SM, et al. Multiplexed echo planar imaging for sub-second whole brain FMRI and fast diffusion imaging. PLoS One. 2010; 5:e15710.

33. Feinberg DA, Setsompop K. Ultra-fast MRI of the human brain with simultaneous multi-slice imaging. J Magn Reson. 2013;229:90-100.

34. Breuer FA, Blaimer M, Heidemann RM, et al. Controlled aliasing in parallel imaging results in higher acceleration (CAIPIRINHA) for multi-slice imaging. Magn Reson Med. 2005;53:684-691.

35. Cauley SF, Polimeni JR, Bhat H, et al. Interslice leakage artifact reduction technique for simultaneous multislice acquisitions. Magn Reson Med. 2014;72: 93-102

36. Hargreaves BA, Cunningham CH, Nishimura DG, et al. Variable-rate selective excitation for rapid MRI sequences. Magn Reson Med. 2004;52:590-597.

37. Auerbach EJ, Xu J, Yacoub E, et al. Multiband accelerated spin-echo echo planar imaging with reduced peak RF power using time-shifted RF pulses. Magn Reson Med. 2013;69:1261-1267.

38. Norris DG, Koopmans PJ, Boyacioglu R, et al. Power Independent of Number of Slices (PINS) radiofrequency pulses for low-power simultaneous multislice excitation. Magn Reson Med. 2011;66:1234-1240.

39. Porter DA, Heidemann RM. High resolution diffusion-weighted imaging using readout-segmented echo-planar imaging, parallel imaging and a two-dimensional navigator-based reacquisition. Magn Reson Med. 2009;62:468-475.

40. Morelli J, Porter D, Ai F, et al. Clinical evaluation of single-shot and readoutsegmented diffusion-weighted imaging in stroke patients at 3 T. Acta Radiol. 2013;54:299-306.

41. Foltz WD, Porter DA, Simeonov A, et al. Readout-segmented echo-planar diffusion-weighted imaging improves geometric performance for image-guided radiation therapy of pelvic tumors. Radiother Oncol. 2015;117:525-531.

42. Frost R, Jezzard P, Douaud G, et al. Scan time reduction for readout-segmented EPI using simultaneous multislice acceleration: Diffusion-weighted imaging at 3 and 7 Tesla. Magn Reson Med. 2014;74:136-149.

43. Runge VM, Richter JK, Klarhöfer M, et al. Simultaneous multi-slice (slice accelerated) diffusion EPI: early experience for brain ischemia and cervical lymphadenopathy. MAGNETOM Flash. 2015;63:92-95.

44. Filli L, Ghafoor S, Kenkel D, et al. Simultaneous multi-slice readout-segmented echo planar imaging for accelerated diffusion-weighted imaging of the breast. Eur J Radiol. 2016;85:274-278.

45. Taviani V, Alley MT, Banerjee S, et al. High-resolution diffusion-weighted imaging of the breast with multiband 2D radiofrequency pulses and a generalized parallel imaging reconstruction. Magn Reson Med. 2016;(on line ahead of print).

46. Hoch MJ, Chung S, Ben-Eliezer N, et al. New clinically feasible 3 T MRI protocol to discriminate internal brain stem anatomy. AJNR Am J Neuroradiol. 2016;37: 1058-1065.

47. Hoge RD, Badhwar A, Doyon J, et al. Improving sensitivity and specificity in BOLD fMRI using simultaneous multi-slice acquistiion. MAGNETOM Flash. 2015;63:65-69.

48. Miller KL, Bartsch AJ, Smith SM. Simultaneous multi-slice imaging for restingstate fMRI. MAGNETOM Flash. 2015;63:70-77.

49. Boyacioglu R, Schulz J, Koopmans PJ, et al. Improved sensitivity and specificity for resting state and task fMRI with multiband multi-echo EPI compared to multiecho EPI at 7 T. Neuroimage. 2015;119:352-361.

50. Van Essen DC, Ugurbil K, Auerbach E, et al. The Human Connectome Project: a data acquisition perspective. Neuroimage. 2012;62:2222-2231.

51. Stab D, Wech T, Breuer FA, et al. High resolution myocardial first-pass perfusion imaging with extended anatomic coverage. J Magn Reson Imaging. 2014;39: 1575-1587.

52. Mekkaoui C, Reese TG, Jackowski MP, et al. Diffusion MRI in the heart. NMR Biomed. 2015. DOI: 10.1002/nbm.3426.

53. Obele CC, Glielmi C, Ream J, et al. Simultaneous multislice accelerated freebreathing diffusion-weighted imaging of the liver at 3 T. Abdom Imaging. 2015; 40:2323-2330.
54. Hennig J, Nauerth A, Friedburg H. RARE imaging: a fast imaging method for clinical MR. Magn Reson Med. 1986;3:823-833.

55. Lustig M, Donoho D, Pauly JM. Sparse MRI: the application of compressed sensing for rapid MR imaging. Magn Reson Med. 2007;58:1182-1195.

56. Liang D, Liu B, Ying L. Accelerating sensitivity encoding using compressed sensing. Conf Proc IEEE Eng Med Biol Soc. 2008:1667-1670.

57. Fushimi Y, Fujimoto K, Okada T, et al. Compressed sensing 3-dimensional timeof-flight magnetic resonance angiography for cerebral aneurysms: optimization and evaluation. Invest Radiol. 2016;51:228-235.

58. Clark Z, Johnson KM, Wu Y, et al. Accelerated time-resolved contrast-enhanced magnetic resonance angiography of dural arteriovenous fistulas using highly constrained reconstruction of sparse cerebrovascular data sets. Invest Radiol. 2016;51:365-371

59. Jung H, Sung K, Nayak KS, et al. k-t FOCUSS: a general compressed sensing framework for high resolution dynamic MRI. Magn Reson Med. 2009;61: 103-116.

60. Ma D, Gulani V, Seiberlich N, et al. Magnetic resonance fingerprinting. Nature 2013;495:187-192.

61. Zijlstra F, Viergever MA, Seevinck PR. Evaluation of variable density and datadriven $\mathrm{k}$-space undersampling for compressed sensing magnetic resonance imaging. Invest Radiol. 2016;51:410-419.

62. Dautry R, Edjlali M, Roca P, et al. Interest of HYPR flow dynamic MRA for characterization of cerebral arteriovenous malformations: comparison with TRICKS MRA and catheter DSA. Eur Radiol. 2015;25:3230-3237.

63. Goebel J, Nensa F, Schemuth HP, et al. Compressed sensing cine imaging with high spatial or high temporal resolution for analysis of left ventricular function. J Magn Reson Imaging. 2016;44:366-374

64. Haubenreisser H, Henzler T, Budjan J, et al. Right ventricular imaging in 25 seconds: evaluating the use of sparse sampling CINE with iterative reconstruction for volumetric analysis of the right ventricle. Invest Radiol. 2016;51: 379-386

65. Forman C, Piccini D, Grimm R, et al. Reduction of respiratory motion artifacts for free-breathing whole-heart coronary MRA by weighted iterative reconstruction. Magn Reson Med. 2015;73:1885-1895.

66. Bhave S, Lingala SG, Newell JD Jr, et al. Blind compressed sensing enables 3-dimensional dynamic free breathing magnetic resonance imaging of lung volumes and diaphragm motion. Invest Radiol. 2016;51:387-399.

67. Zhang T, Cheng JY, Potnick AG, et al. Fast pediatric 3D free-breathing abdominal dynamic contrast enhanced MRI with high spatiotemporal resolution. J Magn Reson Imaging. 2015;41:460-473.

68. Chandarana H, Block TK, Ream J, et al. Estimating liver perfusion from free-breathing continuously acquired dynamic gadolinium-ethoxybenzyldiethylenetriamine pentaacetic acid-enhanced acquisition with compressed sensing reconstruction. Invest Radiol. 2015;50:88-94.

69. Riffel P, Zoellner FG, Budjan J, et al. "One-stop shop": free-breathing dynamic contrast-enhanced magnetic resonance imaging of the kidney using iterative reconstruction and continuous golden-angle radial sampling. Invest Radiol. 2016; 51:714-719.

70. Feng L, Axel L, Chandarana H, et al. XD-GRASP: golden-angle radial MRI with reconstruction of extra motion-state dimensions using compressed sensing. Magn Reson Med. 2016;75:775-788.

71. Chandarana H, Feng L, Ream J, et al. Respiratory motion-resolved compressed sensing reconstruction of free-breathing radial acquisition for dynamic liver magnetic resonance imaging. Invest Radiol. 2015;50:749-756.

72. Chandarana H, Doshi AM, Shanbhogue A, et al. Three-dimensional MR cholangiopancreatography in a breath hold with sparsity-based reconstruction of highly undersampled data. Radiology. 2016;280:585-594.

73. Fritz J, Ahlawat S, Demehri S, et al. Compressed sensing SEMAC: 8-fold accelerated high resolution metal artifact reduction MRI of cobalt-chromium knee arthroplasty implants. Invest Radiol. 2016;51:666-676.

74. Fritz J, Raithel E, Thawait GK, et al. Six-fold acceleration of high-spatial resolution 3D SPACE MRI of the knee through incoherent k-Space undersampling and iterative reconstruction-first experience. Invest Radiol. 2016;51:400-409.

75. Yamamoto T, Fujimoto K, Okada T, et al. Time-of-flight magnetic resonance angiography with sparse undersampling and iterative reconstruction: comparison with conventional parallel imaging for accelerated imaging. Invest Radiol. 2016;51: $372-378$ 